International Journal of Mathematics

(C) World Scientific Publishing Company

\title{
DIRAC STRUCTURES OF OMNI-LIE ALGEBROIDS
}

\author{
ZHUO CHEN \\ Department of Mathematics, Tsinghua University, Beijing 100084, China \\ zchen@math.tsinghua.edu.cn \\ ZHANGJU LIU \\ Department of Mathematics and LMAM, Peking University, Beijing 100871, China \\ liuzj@pku.edu.cn \\ YUNHE SHENG \\ Mathematics School \& Institute of Jilin University, Changchun 130012, Jilin, China \\ shengyh@jlu.edu.cn
}

\begin{abstract}
Omni-Lie algebroids are generalizations of Alan Weinstein's omni-Lie algebras. A Dirac structure in an omni-Lie algebroid $\mathfrak{D} E \oplus \mathfrak{J} E$ is necessarily a Lie algebroid together with a representation on $E$. We study the geometry underlying these Dirac structures in the light of reduction theory. In particular, we prove that there is a one-to-one correspondence between reducible Dirac structures and projective Lie algebroids in $\mathcal{T}=T M \oplus E$; we establish the relation between the normalizer $N_{L}$ of a reducible Dirac structure $L$ and the derivation algebra $\operatorname{Der}(\mathbf{b}(L))$ of the projective Lie algebroid $\mathbf{b}(L)$; we study the cohomology group $\mathrm{H}^{\bullet}\left(L, \rho_{L}\right)$ and the relation between $N_{L}$ and $\mathrm{H}^{1}\left(L, \rho_{L}\right)$; we describe Lie bialgebroids using the adjoint representation; we study the deformation of a Dirac structure $L$, which is related with $\mathrm{H}^{2}\left(L, \rho_{L}\right)$.
\end{abstract}

Keywords: omni-Lie algebroid, Dirac structures, local Lie algebras, reduction, normalizer, deformation

Mathematics Subject Classification 2000: 17B66, 58H05

\section{Introduction}

Lie algebroids (and local Lie algebras in the sense of Kirillov [16]) are generalizations of Lie algebras that naturally appear in Poisson geometry (and its variations, e.g., Jacobi manifolds in the sense of Lichnerowicz [19])(see [23] for a detailed description of this subject). Courant algebroids are combinations of Lie algebroids and quadratic Lie algebras. It was originally introduced in [10] by T. Courant where he first called them Dirac manifolds, and then were re-named after him in 22 (see also an alternate definition [32]) by Liu, Weinstein and Xu to describe the double of a Lie bialgebroid. Recently, several applications of Courant algebroids and Dirac structures have been found in different fields, e.g., Manin pairs and moment maps [15]; generalized complex structures [4]12]; $L_{\infty}$-algebras and symplectic supermanifolds 
[2 24 25]; gerbes [27] as well as BV algebras and topological field theories [14 26 .

Motivated by an integrability problem of the Courant bracket, A. Weinstein gives a linearization of the Courant bracket at a point [36], which had been studied from

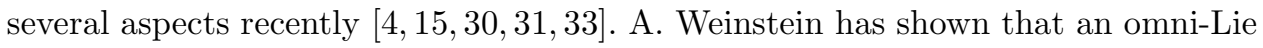
algebra structure can encode all Lie algebra structures on a vector space, the next step is, logically, to find out candidates that could encode all Lie algebroid structures on a vector bundle. In a recent work [7, we have given a definitive answer to this question. Over there, a generalized Courant algebroid structure is defined on the direct sum bundle $\mathfrak{D} E \oplus \mathfrak{J} E$, where $\mathfrak{D} E$ and $\mathfrak{J} E$ are the gauge Lie algebroid and the jet bundle of a vector bundle $E$ respectively. Such a structure is called an omni-Lie algebroid since it reduces to the omni-Lie algebra if the base manifold is a point 36 . Furthermore, an omni-Lie algebroid is the first example of $E$-Courant algebroids [8].

It is well known that the theory of Dirac structures has wide and deep applica-

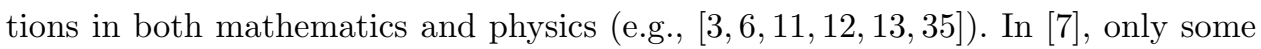
special Dirac structures were studied. The authors proved that there is a one-to-one correspondence between Dirac structures coming from bundle maps $\mathfrak{J} E \rightarrow \mathfrak{D} E$ and Lie algebroid (local Lie algebra) structures on $E$ when $\operatorname{rank}(E) \geq 2$ ( $E$ is a line bundle). In other words, Dirac structures that are graphs of maps actually underlines the geometric objects of Lie algebroids, or local Lie algebras.

As a continuation of [7, the present paper explores what a general Dirac structure of the omni-Lie algebroid can encode. For a vector space $V$, Weinstein proved that Dirac structures in the omni-Lie algebra $\mathfrak{g l}(V) \oplus V$ correspond to Lie algebra structures on subspaces of $V$ in [36. For a vector bundle $E$ over $M$, Dirac structures in the omni-Lie algebroid $\mathcal{E}=\mathfrak{D} E \oplus \mathfrak{J} E$ turn out to be more complicated than that of omni-Lie algebras. The key concept that we need is a projective Lie algebroid, which is a subbundle $A \subset \mathcal{T}=T M \oplus E$, equipped with a Lie algebroid structure such that the anchor is the projection from $A$ to $T M$. A Dirac structure $L \subset \mathcal{E}$ is called reducible if $\mathbf{b}(L)$ is a regular subbundle of $\mathcal{T}$. We will see that any Dirac structure is reducible if $\operatorname{rank}(E) \geq 2$ (Lemma 3.1). The main result is Theorem 3.7 which claims a one-to-one correspondence between reducible Dirac structures in $\mathcal{E}$ and projective Lie algebroids in $\mathcal{T}$. In fact, the projection of a reducible Dirac structure $L$ to $\mathcal{T}$ yields a projective Lie algebroid $\mathbf{b}(L)$ and, conversely, a projective Lie algebroid $A \subset \mathcal{T}$ can be uniquely lifted to a Dirac structure $L^{A}$ using a connection in $E$.

Furthermore, using the falling operator $(\cdot)$, we establish a connection between the derivation algebra $\operatorname{Der}(A)$ of a projective Lie algebroid $A$ and the normalizer $N_{L^{A}}$ of the corresponding lifted Dirac structure $L^{A}$. We prove that, for any $X \in$ $N_{L^{A}}, X_{\bullet} \in \operatorname{Der}(A)$. Conversely, any $\delta \in \operatorname{Der}(A)$ can be lifted to an element in $N_{L^{A}}$. Another observation is that, to any Dirac structure $L \subset \mathcal{E}$, there associates a representation of $L$ on $E$, namely $\rho_{L}: L \longrightarrow \mathfrak{D} E$ (Proposition 2.5). So there is an associated cohomology group $\mathrm{H}^{\bullet}\left(L, \rho_{L}\right)$. We will see that the normalizer of $L$ is related with $\mathrm{H}^{1}\left(L, \rho_{L}\right)$ and the deformation of $L$ is related with $\mathrm{H}^{2}\left(L, \rho_{L}\right)$. 
This paper is organized as follows. In Section 2 we recall the basic properties of omni-Lie algebroids. In Section 3 we state the main result of this paper - the correspondence between reducible Dirac structures and projective Lie algebroids. In Section 4, several interesting examples are discussed. In Section 5 , we study the relation between the normalizer of a reducible Dirac structure and Lie derivations. In Section [6] we give some applications of the related cohomologies of Dirac structures.

\section{Omni-Lie Algebroids}

We use the following convention throughout the paper: $E \rightarrow M$ denotes a vector bundle $E$ over a smooth manifold $M$ (we assume that $E$ is not a zero bundle), d : $\Omega^{\bullet}(M) \rightarrow \Omega^{\bullet+1}(M)$ the usual deRham differential of forms and $m$ an arbitrary point in $M$. By $\mathcal{T}$ we denote the direct sum $T M \oplus E$ and use $p r_{T M}, p r_{E}$, respectively, to denote the projection from $\mathcal{T}$ to $T M$ and $E$.

First, we briefly review the notion of omni-Lie algebroids defined in [7, which generalizes omni-Lie algebras defined by A. Weinstein in [36]. Given a vector bundle $E$, let $\mathfrak{J} E$ be the first jet bundle of $E[28$, and $\mathfrak{D} E$ the gauge Lie algebroid of $E$ [23. These two vector bundles associate, respectively, with the jet sequence:

$$
0 \longrightarrow \operatorname{Hom}(T M, E) \stackrel{\mathbb{e}}{\longrightarrow} \mathfrak{J} E \stackrel{\mathbb{P}}{\longrightarrow} E \rightarrow 0,
$$

and the Atiyah sequence:

$$
0 \longrightarrow \mathfrak{g l}(E) \stackrel{\text { i }}{\longrightarrow} \mathfrak{D} E \stackrel{\alpha}{\longrightarrow} T M \longrightarrow 0 .
$$

The embedding maps $\mathbb{e}$ and $\dot{i}$ in the above two exact sequences will be ignored when there is no risk of confusion. It is well known that $\mathfrak{D} E$ is a transitive Lie algebroid over $M$, with the anchor $\alpha$ as above [17. The $E$-duality between two vector bundles is defined as follows.

Definition 2.1. Let $A, B$ and $E$ be vector bundles over $M$. We say that $B$ is an $E$ dual bundle of $A$ if there is a $C^{\infty}(M)$-bilinear $E$-valued pairing $\langle\cdot, \cdot\rangle_{E}: A \times_{M} B \rightarrow$ $E$ which is nondegenerate, that is, the map $a \mapsto\langle a, \cdot\rangle_{E}$ is an embedding of $A$ into $\operatorname{Hom}(B, E)$, and similarly for the $B$-entry.

An important result in $[7$ is that $\mathfrak{J} E$ is an $E$-dual bundle of $\mathfrak{D} E$ with some nice properties. In fact, we have a nondegenerate $E$-pairing $\langle\cdot, \cdot\rangle_{E}$ between $\mathfrak{J} E$ and $\mathfrak{D} E$ :

$$
\langle\mu, \mathfrak{d}\rangle_{E}=\langle\mathfrak{d}, \mu\rangle_{E} \triangleq \mathfrak{d} u, \quad \forall \mu=[u]_{m} \in \mathfrak{J} E, u \in \Gamma(E), \mathfrak{d} \in \mathfrak{D} E .
$$

Moreover, this pairing is $C^{\infty}(M)$-linear and satisfies the following properties:

$$
\begin{aligned}
\langle\mu, \Phi\rangle_{E} & =\Phi \circ \mathbb{P}(\mu), \quad \forall \Phi \in \mathfrak{g l}(E), \mu \in \mathfrak{J} E ; \\
\langle\mathfrak{y}, \mathfrak{d}\rangle_{E} & =\mathfrak{y} \circ \alpha(\mathfrak{d}), \quad \forall \mathfrak{y} \in \operatorname{Hom}(T M, E), \mathfrak{d} \in \mathfrak{D} E .
\end{aligned}
$$

An equivalent expression is that we can define $\mathfrak{J} E$ by $\mathfrak{D} E$,

$$
\mathfrak{J} E \cong\left\{\nu \in \operatorname{Hom}(\mathfrak{D} E, E) \mid \nu(\Phi)=\Phi \circ \nu\left(\mathbf{1}_{E}\right), \quad \forall \Phi \in \mathfrak{g l}(E)\right\} \subset \operatorname{Hom}(\mathfrak{D} E, E) .
$$


Conversely, $\mathfrak{D} E$ is also determined by $\mathfrak{J} E$ :

$\mathfrak{D} E \cong\{\delta \in \operatorname{Hom}(\mathfrak{J} E, E) \mid \exists x \in T M$, s.t. $\delta(\mathfrak{y})=\mathfrak{y}(x), \quad \forall \mathfrak{y} \in \operatorname{Hom}(T M, E)\}$

For a Lie algebroid $(\mathcal{A},[\cdot, \cdot], \alpha)$ over $M$, a representation of $\mathcal{A}$ on a vector bundle $E \rightarrow M$ is a Lie algebroid morphism $\mathscr{L}: \mathcal{A} \rightarrow \mathfrak{D} E$. We may also refer to $E$ as an $\mathcal{A}$-module. To such a representation, there associates a cochain complex $\sum_{i \geq 0} \Omega^{i}(\mathcal{A}, E)=\sum_{i \geq 0} \Gamma\left(\operatorname{Hom}\left(\wedge^{i} \mathcal{A}, E\right)\right)$ with the coboundary operator:

$$
\mathrm{d}_{\mathcal{A}}: \Omega^{\bullet}(\mathcal{A}, E) \rightarrow \Omega^{\bullet+1}(\mathcal{A}, E)
$$

defined in a similar fashion as that of the deRham differential 23 . Since $\mathfrak{D} E$ is a Lie algebroid and $E$ is a natural $\mathfrak{D} E$-module, we have the cochain complex:

$$
\Omega^{\bullet}(\mathfrak{D} E, E)=\Gamma\left(\operatorname{Hom}\left(\wedge^{\bullet} \mathfrak{D} E, E\right)\right)
$$

with the coboundary operator:

$$
\mathbb{d}: \Omega^{\bullet}(\mathfrak{D} E, E) \rightarrow \Omega^{\bullet+1}(\mathfrak{D} E, E) .
$$

Note that, $\forall u \in \Gamma(E), \mathbb{d} u \in \Omega^{1}(\mathfrak{D} E, E)$ is a section of $\mathfrak{J} E$ and we have a formula:

$$
\mathbb{d}(f u)=f \mathbb{d} u+\mathrm{d} f \otimes u, \quad \forall f \in C^{\infty}(M), u \in \Gamma(E) .
$$

The section space $\Gamma(\mathfrak{J} E)$ is an invariant subspace of the Lie derivative $\mathfrak{L}_{\mathfrak{d}}$ for any $\mathfrak{d} \in \Gamma(\mathfrak{D} E)$. Here $\mathfrak{L}_{\mathfrak{d}}$ is defined by the Leibniz rule as follows:

$$
\left\langle\mathfrak{L}_{\mathfrak{d}} \mu, \mathfrak{d}^{\prime}\right\rangle_{E} \triangleq \mathfrak{d}\left\langle\mu, \mathfrak{d}^{\prime}\right\rangle_{E}-\left\langle\mu,\left[\mathfrak{d}, \mathfrak{d}^{\prime}\right]_{\mathfrak{D}}\right\rangle_{E}, \quad \forall \mu \in \Gamma(\mathfrak{J} E), \quad \mathfrak{d}^{\prime} \in \Gamma(\mathfrak{D} E) .
$$

Definition 2.2. 7] We call the quadruple $\left(\mathcal{E},\{\cdot, \cdot\},(\cdot, \cdot)_{E}, \rho\right)$ an omni-Lie algebroid, where $\mathcal{E}=\mathfrak{D} E \oplus \mathfrak{J} E, \rho$ is the projection from $\mathcal{E}$ to $\mathfrak{D} E$, the bracket $\{\cdot, \cdot\}: \Gamma(\mathcal{E}) \times \Gamma(\mathcal{E}) \longrightarrow \Gamma(\mathcal{E})$ is defined by

$$
\{\mathfrak{d}+\mu, \mathfrak{r}+\nu\} \triangleq[\mathfrak{d}, \mathfrak{r}]_{\mathfrak{D}}+\mathfrak{L}_{\mathfrak{d}} \nu-\mathfrak{L}_{\mathfrak{r}} \mu+\mathbb{d}\langle\mu, \mathfrak{r}\rangle_{E},
$$

and $(\cdot, \cdot)_{E}$ is a nondegenerate symmetric $E$-valued 2 -form on $\mathcal{E}$ defined by:

$$
(\mathfrak{d}+\mu, \mathfrak{r}+\nu)_{E} \triangleq \frac{1}{2}\left(\langle\mathfrak{d}, \nu\rangle_{E}+\langle\mathfrak{r}, \mu\rangle_{E}\right),
$$

for any $\mathfrak{d}, \mathfrak{r} \in \mathfrak{D} E, \mu, \nu \in \mathfrak{J} E$.

Theorem 2.3. 7 An omni-Lie algebroid satisfies the following properties:

1) $(\Gamma(\mathcal{E}),\{\cdot, \cdot\})$ is a Leibniz algebra,

2) $\rho\{X, Y\}=[\rho(X), \rho(Y)]_{\mathfrak{D}}$,

3) $\{X, f Y\}=f\{X, Y\}+(\alpha \circ \rho(X))(f) Y$,

4) $\{X, X\}=\mathbb{d}(X, X)_{E}$,

5) $\rho(X)(Y, Z)_{E}=(\{X, Y\}, Z)_{E}+(Y,\{X, Z\})_{E}$, 
for any $X, Y, Z \in \Gamma(\mathcal{E})$ and $f \in C^{\infty}(M)$.

It is easy to obtain the following equalities:

$$
\begin{aligned}
\{f X, Y\} & =f\{X, Y\}-(\alpha \circ \rho(Y))(f) Y+2 \mathrm{~d} f \otimes(X, Y)_{E}, \\
\{X, Y\}+\{Y, X\} & =2 \mathbb{d}(X, Y)_{E} .
\end{aligned}
$$

For a subbundle $S \subset \mathcal{E}$, we denote

$$
S^{\perp}=\left\{X \in \mathcal{E} \mid(X, s)_{E}=0, \quad \forall s \in S\right\} .
$$

We call $S$ isotropic with respect to $(\cdot, \cdot)_{E}$ if $S \subset S^{\perp}$.

Definition 2.4. 7] A Dirac structure in the omni-Lie algebroid $\mathcal{E}$ is a maximal isotropid subbundle $L \subset \mathcal{E}$ such that $\{\Gamma(L), \Gamma(L)\} \subset \Gamma(L)$.

Proposition 2.5. 7 A Dirac structure $L$ is necessarily a Lie algebroid with the restricted bracket and the anchor $\alpha \circ \rho$. Moreover, $\rho_{L}=\left.\rho\right|_{L}: L \rightarrow \mathfrak{D} E$ is a representation of $L$ on $E$.

For $\mathcal{T}=T M \oplus E$, we have the standard decomposition

$$
\operatorname{Hom}(\mathcal{T}, E)=\mathfrak{g l}(E) \oplus \operatorname{Hom}(T M, E) .
$$

The following exact sequence will be referred as the omni-sequence of $E$.

$$
0 \rightarrow \operatorname{Hom}(\mathcal{T}, E) \stackrel{\mathrm{a}}{\longrightarrow} \mathcal{E} \stackrel{\mathrm{b}}{\longrightarrow} \mathcal{T} \rightarrow 0,
$$

where the maps $\mathbf{a}$ and $\mathbf{b}$ are defined, respectively, by

$$
\begin{aligned}
& \mathbf{a}(\Phi+\mathfrak{y})=\dot{\mathfrak{i}}(\Phi)+\mathbb{e}(\mathfrak{y}), \quad \forall \Phi \in \mathfrak{g l}(E), \mathfrak{y} \in \operatorname{Hom}(T M, E), \\
& \mathbf{b}(\mathfrak{d}+\mu)=\alpha(\mathfrak{d})+\mathfrak{p}(\mu), \quad \forall \mathfrak{d} \in \mathfrak{D} E, \mu \in \mathfrak{J} E .
\end{aligned}
$$

We regard $\operatorname{Hom}(\mathcal{T}, E)$ as a subbundle of $\mathcal{E}$ and omit the embedding a. Evidently, $\operatorname{Hom}(\mathcal{T}, E)$ is a maximal isotropic subbundle of $\mathcal{E}$. In fact, it is a Dirac structure of $\mathcal{E}$ and the bracket is given by

$$
\{\alpha, \beta\}=\alpha \circ \beta-\beta \circ \alpha, \quad \forall \alpha, \beta \in \Gamma(\operatorname{Hom}(\mathcal{T}, E)) .
$$

In particular, if $\alpha=\Phi+\phi, \beta=\Psi+\psi$, where $\Phi, \Psi \in \Gamma(\mathfrak{g l}(E)), \phi, \psi \in$ $\Gamma(\operatorname{Hom}(T M, E))$, then

$$
\{\Phi, \Psi\}=\Phi \circ \Psi-\Psi \circ \Phi, \quad\{\phi, \psi\}=0, \quad\{\Phi, \phi\}=\Phi \circ \phi .
$$

\section{Lemma 2.6.}

(1) The subspace $\Gamma(\operatorname{Hom}(\mathcal{T}, E))$ is a right ideal of $\Gamma(\mathcal{E})$.

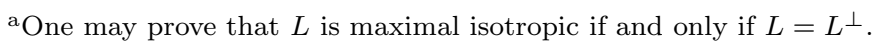


6 Z. Chen, Z. Liu and Y. Sheng

(2) For any $h \in \Gamma(\operatorname{Hom}(\mathcal{T}, E)), X \in \Gamma(\mathcal{E})$, we have

$$
\mathbf{b}\{h, X\}=h(\mathbf{b}(X)) .
$$

Proof. For any $X=\mathfrak{d}+\mu \in \Gamma(\mathcal{E})$ and $h=\Phi+\mathfrak{y} \in \Gamma(\operatorname{Hom}(\mathcal{T}, E))$, we have

$$
\{\mathfrak{d}+\mu, \Phi+\mathfrak{y}\}=[\mathfrak{d}, \Phi]_{\mathfrak{D}}+\mathfrak{L}_{\mathfrak{d}} \eta-\mathfrak{L}_{\Phi} \mu+\mathbb{d}\langle\mu, \Phi\rangle_{E} .
$$

Since

$$
\mathbb{p}\left(-\mathfrak{L}_{\Phi} \mu+\mathbb{d}\langle\mu, \Phi\rangle_{E}\right)=-\Phi \mathbb{p}(\mu)+\langle\mu, \Phi\rangle_{E}=0
$$

and $\alpha[\mathfrak{d}, \Phi]_{\mathfrak{D}}=0$, we have

$$
\{\mathfrak{d}+\mu, \Phi+\mathfrak{y}\} \in \Gamma(\operatorname{Hom}(\mathcal{T}, E)),
$$

which implies that $\Gamma(\operatorname{Hom}(\mathcal{T}, E))$ is a right ideal of $\Gamma(\mathcal{E})$.

On the other hand, we have

$$
\begin{aligned}
\mathbf{b}\{h, X\} & =\mathbf{b}\left([\Phi, \mathfrak{d}]_{\mathfrak{D}}+\mathfrak{L}_{\Phi} \mu-\mathfrak{L}_{\mathfrak{d}} \mathfrak{y}+\mathbb{d}\langle\mathfrak{d}, \eta\rangle_{E}\right) \\
& =\Phi(\mathfrak{p} \mu)+\mathfrak{y}(\alpha \mathfrak{d})=h(\mathbf{b}(X)),
\end{aligned}
$$

which completes the proof.

\section{Dirac Structures and Their Reductions}

Let us first study some basic properties of maximal isotropic subbundles of $\mathcal{E}$. For any subbundle $Q \subset \mathcal{T}$, define:

$$
Q^{0} \triangleq\{h \in \operatorname{Hom}(\mathcal{T}, E) \mid h(Q)=0\} .
$$

Lemma 3.1. If $\operatorname{rank}(E)=r, \operatorname{dim}(M)=d$, then for any maximal isotropic subbundle $L \subset \mathcal{E}$, we have

$$
\operatorname{rank}\left(L_{m}\right)=(1-r) \operatorname{rank}\left(\mathbf{b}\left(L_{m}\right)\right)+r(d+r), \quad \forall m \in M .
$$

Consequently, if $r \geq 2$, both $\mathbf{b}(L)$ and $\mathbf{b}(L)^{0}$ are regular subbundles of, respectively, $\mathcal{T}$ and $\mathcal{E}$. If $r=1$, that is, $E$ is a line bundle, then $\operatorname{rank}(L)=d+1$.

Proof. Since $L$ is maximal isotropic, or equivalently, $L=L^{\perp}$, it is not hard to establish the following exact sequence:

$$
0 \longrightarrow\left(\mathbf{b}\left(L_{m}\right)\right)^{0} \stackrel{\mathbf{a}}{\longrightarrow} L_{m} \longrightarrow \text { b } \mathrm{b}\left(L_{m}\right) \longrightarrow 0 .
$$

Therefore, we have

$$
\begin{aligned}
\operatorname{rank}\left(L_{m}\right) & =\operatorname{rank}\left(\mathbf{b}\left(L_{m}\right)\right)+\operatorname{rank}\left(\mathbf{b}\left(L_{m}\right)\right)^{0} \\
& =\operatorname{rank}\left(\mathbf{b}\left(L_{m}\right)\right)+\left(r+d-\operatorname{rank}\left(\mathbf{b}\left(L_{m}\right)\right)\right) \times r \\
& =(1-r) \operatorname{rank}\left(\mathbf{b}\left(L_{m}\right)\right)+r(d+r) .
\end{aligned}
$$


Definition 3.2. For a vector subbundle $A \subset \mathcal{T}$, a section $s: A \longrightarrow \mathcal{E}$ (i.e. $\left.\mathbf{b} \circ s=\mathbf{1}_{A}\right)$ is called isotropic if its image $s(A) \subset \mathcal{E}$ is isotropic. Two isotropic sections $s_{1}$ and $s_{2}$ are said to be equivalent if $\left(s_{1}-s_{2}\right)(A) \subset A^{0}$. The equivalence class of an isotropic section $s$ is denoted by $\widetilde{s}$.

Proposition 3.3. If $\operatorname{rank} E \geq 2$, there is a one-to-one correspondence between maximal isotropic subbundles $L \subset \mathcal{E}$ and pairs $(A, \widetilde{s})$, where $A$ is a subbundle of $\mathcal{T}$ and $s: A \rightarrow \mathcal{E}$ is an isotropic section.

For this reason, we call $(A, \widetilde{s})$ the characteristic pair of $L$, and write $L=L_{s, A}$. Proof. Let $L \subset \mathcal{E}$ be a maximal isotropic subbundle and $A=\mathbf{b}(L)$. By Lemma 3.1. $A$ is a regular subbundle. Any split $s: A \rightarrow L$ of the corresponding exact sequence (3.2) yields an isotropic section and $(A, \widetilde{s})$ is defined to be the characteristic pair of $L$. It is well defined since for any two isotopic sections $s_{1}, s_{2}$, we have $\operatorname{Im}\left(s_{1}-s_{2}\right) \subset \mathbf{b}(L)^{0}=A^{0}$, which is equivalent to $\widetilde{s_{1}}=\widetilde{s_{2}}$.

Conversely, given a subbundle $A \subset \mathcal{T}$ and any characteristic pair $(A, \widetilde{s})$, set $L_{s, A}=s(A) \oplus A^{0}$. Evidently, $L_{s, A}$ is a maximal isotropic subbundle of $\mathcal{E}$ whose characteristic pair is $(A, \widetilde{s})$. It is also clear that if $\widetilde{s_{1}}=\widetilde{s_{2}}, L_{s_{1}, A}=L_{s_{2}, A}$.

One may check that these two constructions are inverse to each other.

Definition 3.4. A projective Lie algebroid is a subbundle $A \subset T M \oplus E$ which is a Lie algebroid $\left(A,[\cdot, \cdot]_{A}, \rho_{A}\right)$ and the anchor $\rho_{A}=\left.p r_{T M}\right|_{A}$.

Example 3.5. Let $\mathcal{A} \longrightarrow N$ be a Lie algebroid over a smooth manifold $N$ and $\alpha$ its anchor. Let $f: M \longrightarrow N$ be a smooth map and $f^{*} \mathcal{A} \rightarrow M$ the pull back bundle along $f$. We denote the pull back Lie algebroid of $\mathcal{A}$ over $M$ by $f^{!} A=T M \oplus_{T N} \mathcal{A}$, which is given by

$$
T M \oplus_{T N} \mathcal{A}=\left\{(x, X) \in T_{m} M \oplus \mathcal{A}_{f(m)} \mid m \in M, \text { and } f_{*}(x)=\alpha(X)\right\} .
$$

Sections of $T M \oplus_{T N} \mathcal{A}$ are of the form:

$$
x \oplus\left(\sum u_{i} \otimes X_{i}\right), \quad x \in \mathfrak{X}(M), u_{i} \in C^{\infty}(M), X_{i} \in \Gamma(\mathcal{A}),
$$

such that $f_{*}(x(m))=\sum u_{i}(m) \alpha\left(X_{i}(f(m))\right)$. The anchor $\alpha^{!}$of the Lie algebroid $f^{!} \mathcal{A}$ is the projection to the first summand. The Lie bracket can be locally expressed by

$$
\begin{aligned}
& {\left[x \oplus\left(\sum u_{i} \otimes X_{i}\right), y \oplus\left(\sum v_{j} \otimes Y_{j}\right)\right] } \\
= & {[x, y] \oplus\left(\sum u_{i} v_{j} \otimes\left[X_{i}, Y_{j}\right]+\sum x\left(v_{j}\right) \otimes Y_{j}-\sum y\left(u_{i}\right) \otimes X_{i}\right) . }
\end{aligned}
$$

Thus the pull back Lie algebroid $f^{!} \mathcal{A}$ of the Lie algebroid $\mathcal{A}$ is a projective Lie algebroid in $T M \oplus f^{*} \mathcal{A}$.

Example 3.6. We suppose that the base manifold $M$ is compact and let $H \subset T M$ be an integrable distribution. It is well known that there is some vector bundle $E$ such that the vector bundle $F=H \oplus E$ is trivial. Suppose that $\operatorname{rank} F=n$ and $\varepsilon_{1}, \cdots, \varepsilon_{n}$ are everywhere linear independent sections of $F$, i.e. a frame of $\Gamma(F)$. 
Write $\varepsilon_{i}=x_{i}+e_{i}$, where $x_{i}$ and $e_{i}$ are sections of $H$ and $E$ respectively. It is clear that $\Gamma(H)=\operatorname{span}\left\{x_{1}, \cdots, x_{n}\right\}$ and $\Gamma(E)=\operatorname{span}\left\{e_{1}, \cdots, e_{n}\right\}$ (over $C^{\infty}(M)$ ). Since $H$ is an integrable distribution, there exist functions $c_{i, j}^{k} \in C^{\infty}(M)$ such that $\left[x_{i}, x_{j}\right]=c_{i, j}^{k} x_{k}$. Now set $\left[\varepsilon_{i}, \varepsilon_{j}\right]=c_{i, j}^{k} \varepsilon_{k}$. It is easy to see that $F$ is a projective Lie algebroid in $T M \oplus E$.

A Dirac structure $L \subset \mathcal{E}$ is called reducible if $\mathbf{b}(L)$ is a regular subbundle of $\mathcal{T}$. By Lemma 3.1, any Dirac structure is reducible if $\operatorname{rank}(E) \geq 2$. As a main result of this paper, the following theorem describes the nature of reducible Dirac structures in the omni-Lie algebroid $\mathcal{E}$.

Theorem 3.7. For any vector bundle $E$, there is a one-to-one correspondence between reducible Dirac structures $L \subset \mathcal{E}$ and projective Lie algebroids $A=\mathbf{b}(L) \subset \mathcal{T}$ such that $A$ is the quotient Lie algebroid of $L$.

Proof. Assume that $L$ is a reducible Dirac structure and let $A=\mathbf{b}(L) \subset \mathcal{T}$. Then we have the following exact sequence:

$$
0 \longrightarrow A^{0} \stackrel{\mathbf{a}}{\longrightarrow} L \stackrel{\mathbf{b}}{\longrightarrow} A \longrightarrow 0 .
$$

By $L$ being reducible, $A$ is a regular subbundle, $A^{0}$ as well. The anchor $\alpha \circ \rho$ vanishes if restricted on $A^{0}$. Furthermore, by Lemma 2.6 and the fact that $L$ is a Dirac structure, $A^{0}$ is an ideal of $L$. So we have a quotient Lie algebroid structure $\left(A,[\cdot, \cdot]_{A}, \rho_{A}\right)$, where $\rho_{A}$ is clearly the projection to $T M$. This proves that $A$ is indeed a projective Lie algebroid.

Conversely, for the projective Lie algebroid $\left(A,[\cdot, \cdot]_{A}, \rho_{A}\right)$, define a subset $L^{A} \subset$ $\mathbf{b}^{-1}(A) \subset \mathcal{E}$ by:

$$
\begin{gathered}
L_{m}^{A} \triangleq\left\{X \in \mathbf{b}^{-1}(A)_{m} \mid \text { for some } \widetilde{X} \in \Gamma\left(\mathbf{b}^{-1}(A)\right) \text { with } \widetilde{X}_{m}=X, \quad\right. \text { there holds } \\
\left.\mathbf{b}\{\widetilde{X}, Y\}_{m}=\left([\mathbf{b} \widetilde{X}, \mathbf{b} Y]_{A}\right)_{m}, \quad \forall Y \in \Gamma\left(\mathbf{b}^{-1}(A)\right)\right\} .
\end{gathered}
$$

Note that by Equation (2.4), we have

$$
\mathbf{b}\{f \widetilde{X}, Y\}_{m}-\left([f \mathbf{b} \tilde{X}, \mathbf{b} Y]_{A}\right)_{m}=f\left(\mathbf{b}\{\tilde{X}, Y\}_{m}-\left([\mathbf{b} \tilde{X}, \mathbf{b} Y]_{A}\right)_{m}\right) .
$$

Hence the above definition does not depend on the choice of $\widetilde{X}$.

To prove that $L^{A}$ is the unique reducible Dirac structure such that the induced projective Lie algebroid is $\left(A,[\cdot, \cdot]_{A}, \rho_{A}\right)$, we need three steps as follows. Step 1 proves that $L^{A}$ is a maximal isotropic subbundle such that $\mathbf{b}\left(L^{A}\right)=A$. Step 2 proves that $L^{A}$ is closed under the bracket $\{\cdot, \cdot\}$ and it follows that $L^{A}$ is a reducible Dirac structure such that the induced projective Lie algebroid is $\left(A,[\cdot, \cdot]_{A}, \rho_{A}\right)$. The last step proves the uniqueness of such Dirac structures.

Step 1. We prove that $L^{A}$ is a maximal isotropic subbundle. We will construct a maximal isotropic subbundle $L_{s_{\gamma}, A}$ using a connection $\gamma$ in the vector bundle $E$ and prove that $L_{s_{\gamma}, A}=L^{A}$. 
Recall that a connection in $E$ is a bundle map $\gamma: T M \rightarrow \mathfrak{D} E$ such that $\alpha \circ \gamma=\mathbf{1}_{T M}$. Associated with $\gamma$ there is a back connection $\omega: \mathfrak{D} E \rightarrow \mathfrak{g l}(E)$, such that $\dot{\text { i }} \circ \omega+\gamma \circ \alpha=\mathbf{1}_{\mathfrak{D} E}$. So we can define a bundle map $\widetilde{\gamma}: E \rightarrow \mathfrak{J} E$ by

$$
\langle\widetilde{\gamma}(e), \mathfrak{d}\rangle_{E} \triangleq \omega(\mathfrak{d})(e)=(\mathfrak{d}-\gamma \circ \alpha(\mathfrak{d}))(e), \quad \forall \mathfrak{d} \in \mathfrak{D} E
$$

such that $\mathrm{p} \circ \widetilde{\gamma}=\mathbf{1}_{E}$. In turn, we get a map:

$$
\gamma+\widetilde{\gamma}: \mathcal{T} \rightarrow \mathcal{E} \quad \text { such that } \quad \mathbf{b} \circ(\gamma+\widetilde{\gamma})=\mathbf{1}_{\mathcal{T}} .
$$

We still denote this map by $\gamma$. This does not make any confusion since it depends on what is put right after it.

Choose an arbitrary subbundle $C \subset \mathcal{T}$, such that $\mathcal{T}=A \oplus C$. Define a bundle $\operatorname{map} \Omega_{\gamma}: \mathcal{T} \wedge \mathcal{T} \rightarrow E$ by

$$
\begin{aligned}
\Omega_{\gamma}(a, b) & =[a, b]_{A}-\mathbf{b}\{\gamma(a), \gamma(b)\}, \quad \forall a, b \in \Gamma(A), \\
\Omega_{\gamma}(c, t) & =0, \quad \forall c \in C, t \in \mathcal{T} .
\end{aligned}
$$

To see that $\Omega_{\gamma} \in \operatorname{Hom}\left(\wedge^{2} \mathcal{T}, E\right)$, first for any $a=x+u, b=y+v \in \Gamma(A)$, where $x, y \in \mathfrak{X}(M), u, v \in \Gamma(E)$, we have

$$
\begin{aligned}
\mathbf{b}\{\gamma(x+u), \gamma(y+v)\} & =\mathbf{b}\left([\gamma(x), \gamma(y)]_{\mathfrak{D}}+\mathfrak{L}_{\gamma(x)} \gamma(v)-\mathfrak{L}_{\gamma(y)} \gamma(u)+\mathbb{d}\langle\gamma(y), \gamma(u)\rangle_{E}\right) \\
& =[\alpha \gamma(x), \alpha \gamma(x)]_{\mathfrak{D}}+\gamma(x)(\mathbb{p} \gamma(v))-\gamma(y)(\mathbb{p} \gamma(u)) \\
& =[x, y]+\gamma(x) v-\gamma(y) u,
\end{aligned}
$$

which implies that

$$
\Omega_{\gamma}(x+u, y+v)=\left([x+u, y+v]_{A}-[x, y]\right)-\gamma(x)(v)+\gamma(y)(u) .
$$

Thus we have $\Omega_{\gamma}(x+u, y+v) \in \Gamma(E)$. On the other hand, for any $f \in C^{\infty}(M)$, we have

$$
\begin{aligned}
\Omega_{\gamma}(x+u, f(y+v)) & =\left([x+u, f(y+v)]_{A}-[x, f y]\right)-\gamma(x)(f v)+\gamma(f y)(u) \\
& =f \Omega_{\gamma}(x+u, y+v)+x(f)(y+v)-x(f) y-\alpha(\gamma(x))(f) v \\
& =f \Omega_{\gamma}(x+u, y+v) .
\end{aligned}
$$

By (3.7) and (3.8), we obtain that $\Omega_{\gamma} \in \operatorname{Hom}\left(\wedge^{2} \mathcal{T}, E\right)$. We also denote the associated skew-symmetric map from $\mathcal{T}$ to $\operatorname{Hom}(\mathcal{T}, E)$ by $\Omega_{\gamma}$.

Define an isotropic section $s_{\gamma}: A \longrightarrow \mathcal{E}$ by

$$
s_{\gamma}(a)=\gamma(a)+\Omega_{\gamma}(a), \quad \forall a \in A .
$$

In fact, for $a=x+u, b=y+v \in \Gamma(A)$, we have

$$
\begin{aligned}
& \left(s_{\gamma}(x+u), s_{\gamma}(y+v)\right)_{E} \\
= & \left(\gamma(x)+\gamma(u)+\Omega_{\gamma}(a), \gamma(y)+\gamma(v)+\Omega_{\gamma}(b)\right)_{E} \\
= & \frac{1}{2}\left(\Omega_{\gamma}(y+v, x+u)+\Omega_{\gamma}(x+u, y+v)+\langle\gamma(x), \gamma(v)\rangle_{E}+\langle\gamma(y), \gamma(u)\rangle_{E}\right)=0 .
\end{aligned}
$$

By Proposition 3.3. we get a maximal isotropic subbundle $L_{s_{\gamma}, A}$ :

$$
L_{s_{\gamma}, A}=\gamma(A)+\Omega_{\gamma}(A)+A^{0} .
$$


We can directly check that $L_{s_{\gamma}, A}$ does not depend on the choice of the connection $\gamma$ and the subbundle $C$. An alternate approach is to prove that $L_{s_{\gamma}, A}=L^{A}$, since $L^{A}$ does not depend on $s_{\gamma}$ and $A$.

Now we prove $L_{s_{\gamma}, A}=L^{A}$. Any $X \in \Gamma\left(L_{s_{\gamma}, A}\right)$ has the form $X=\gamma(a)+\Omega_{\gamma}(a)+h$, where $a=x+u \in \Gamma(A)$ and $h \in \Gamma\left(A^{0}\right)$. For any $Y=\mathfrak{d}+\mu \in \Gamma\left(\mathbf{b}^{-1}(A)\right)$ satisfying $\mathbf{b}(Y)=y+v \in \Gamma(A)$, we have

$$
\begin{aligned}
\mathbf{b}\{X, Y\} & =\mathbf{b}\left(\{\gamma(x)+\gamma(u), \mathfrak{d}+\mu\}+\left\{\Omega_{\gamma}(a)+h, Y\right\}\right) \\
& =\mathbf{b}\left([\gamma(x), \mathfrak{d}]_{\mathfrak{D}}+\mathfrak{L}_{\gamma(x)} \mu-\mathfrak{L}_{\mathfrak{d}} \gamma(u)+\mathbb{d}\langle\gamma(u), \mathfrak{d}\rangle_{E}\right)+\left(\Omega_{\gamma}(a)+h\right)(\mathbf{b}(Y)) \\
& =[x, \alpha \mathfrak{d}]+\gamma(x)(v)-\mathfrak{d}(u)+\langle\gamma(u), \mathfrak{d}\rangle_{E}+\Omega_{\gamma}(x+u, y+v) \\
& =[x, y]+\gamma(x) v-\gamma(y) u+\Omega_{\gamma}(x+u, y+v) \\
& =[x+u, y+v]_{A}, \quad(\operatorname{using}(3.7)) \\
& =[\mathbf{b}(X), \mathbf{b}(Y)]_{A} .
\end{aligned}
$$

Thus, $X \in \Gamma\left(L^{A}\right)$. So we have $L_{s_{\gamma}, A} \subset L^{A}$. Since $\mathbf{b}\left(L^{A}\right) \subset A$, any $X \in L^{A}$ can be written as $X=X_{0}+h$, where $X_{0} \in L_{s_{\gamma}, A}$ and $h \in \operatorname{Hom}(\mathcal{T}, E)$. Thus $h=X-X_{0} \in L^{A} \cap \operatorname{Hom}(\mathcal{T}, E)$.

For any $k \in \operatorname{Hom}\left(\mathcal{T}_{m}, E_{m}\right)=\operatorname{Kerb}$ and $\widetilde{k} \in \Gamma(\operatorname{Hom}(\mathcal{T}, E))$ satisfying $\widetilde{k}(m)=k$, $\forall Y \in \Gamma\left(\mathbf{b}^{-1}(A)\right)$, we have, by Equation (2.7)

$$
\mathbf{b}\{\widetilde{k}, Y\}_{m}-\left([\mathbf{b}(\widetilde{k}), \mathbf{b}(Y)]_{A}\right)_{m}=k(\mathbf{b}(Y)) .
$$

Thus $k \in L_{m}^{A} \cap \operatorname{Hom}\left(\mathcal{T}_{m}, E_{m}\right)$ if and only if $k \in A_{m}^{0}$, that is,

$$
L^{A} \cap \operatorname{Hom}(\mathcal{T}, E)=A^{0} .
$$

So we have proved that $L^{A} \subset L_{s_{\gamma}, A}$. By maximality, $L^{A}=L_{s_{\gamma}, A}$ and hence $L^{A}$ is a maximal isotropic subbundle of $\mathcal{E}$.

Step 2. We prove that $\Gamma\left(L^{A}\right)$ is closed under the bracket operation $\{\cdot, \cdot\}$ and it follows that $L^{A}=L_{s_{\gamma}, A}$ is a reducible Dirac structure.

For any $X_{1}, X_{2} \in \Gamma\left(L^{A}\right)$ and $Y \in \Gamma\left(\mathbf{b}^{-1}(A)\right)$, we have $\left\{X_{1}, X_{2}\right\} \in \Gamma\left(\mathbf{b}^{-1}(A)\right)$ and $\left\{X_{i}, Y\right\} \in \Gamma\left(\mathbf{b}^{-1}(A)\right)$. Moreover, we have

$$
\begin{aligned}
\mathbf{b}\left\{\left\{X_{1}, X_{2}\right\}, Y\right\} & =\mathbf{b}\left\{X_{1},\left\{X_{2}, Y\right\}\right\}-\mathbf{b}\left\{X_{2},\left\{X_{1}, Y\right\}\right\} \\
& =\left[\mathbf{b} X_{1}, \mathbf{b}\left\{X_{2}, Y\right\}\right]_{A}-\left[\mathbf{b} X_{2}, \mathbf{b}\left\{X_{1}, Y\right\}\right]_{A} \\
& =\left[\mathbf{b} X_{1},\left[\mathbf{b} X_{2}, Y\right]_{A}\right]_{A}-\left[\mathbf{b} X_{2},\left[\mathbf{b} X_{1}, Y\right]_{A}\right]_{A} \\
& =\left[\left[\mathbf{b} X_{1}, \mathbf{b} X_{2}\right]_{A}, \mathbf{b} Y\right]_{A} \\
& =\left[\mathbf{b}\left\{X_{1}, X_{2}\right\}, \mathbf{b} Y\right]_{A},
\end{aligned}
$$

which implies that $\left\{X_{1}, X_{2}\right\} \in \Gamma\left(L^{A}\right)$. So $L^{A}$ is a Dirac structure. In Step 1, we have proved that $\mathbf{b}\left(L^{A}\right)=A$, and in turn, $L^{A}$ is a reducible Dirac structure. By definition, the induced projective Lie algebroid is exactly $\left(A,[\cdot, \cdot]_{A}, \rho_{A}\right)$.

Step 3. We prove the uniqueness of such Dirac structures.

Assume that $L^{\prime}$ is another reducible Dirac structure satisfying the same requirements. It suffices to prove that $L^{\prime} \subset L^{A}$, since $L^{A}$ is a maximal isotropic 
subbundle. For any $X \in L_{m}^{\prime}$ and $\tilde{X} \in \Gamma\left(L^{\prime}\right)$ such that $\widetilde{X}_{m}=X$, we prove that $X \in L_{m}^{A}$. In fact, $\forall Y \in \Gamma\left(\mathbf{b}^{-1}(A)\right)$, we are able to find some $Y^{\prime} \in \Gamma\left(L^{\prime}\right)$ such that $\mathbf{b} Y^{\prime}=\mathbf{b} Y$. So we can write $Y=Y^{\prime}+K$, where $K \in \Gamma(\operatorname{Hom}(\mathcal{T}, E))$. By Lemma 2.6. $\{\tilde{X}, K\} \in \Gamma(\operatorname{Hom}(\mathcal{T}, E))$. Thus,

$$
\mathbf{b}\{\tilde{X}, Y\}=\mathbf{b}\left\{\tilde{X}, Y^{\prime}\right\}+\mathbf{b}\{\widetilde{X}, K\}=\left[\mathbf{b} \widetilde{X}, \mathbf{b} Y^{\prime}\right]_{A}=[\mathbf{b} \widetilde{X}, \mathbf{b} Y]_{A},
$$

which implies that $X \in L_{m}^{A}$. So we have $L^{\prime} \subset L^{A}$. The proof of Theorem 3.7 is thus completed.

The projective Lie algebroid $\mathbf{b}(L)$ is called the reduction of the reducible Dirac structure $L$. The reducible Dirac structure $L^{A}$ is called the lift of the projective Lie algebroid $A$.

\section{Some Examples}

Bellow we give some basic examples of Dirac structures in the omni-Lie algebroid.

Example 4.1. For a vector space $V$, our theorem claims a one-to-one correspondence between Dirac structures of the omni-Lie algebra $\mathfrak{g l}(V) \oplus V$ and Lie algebra structures on subspaces of $V$. Thus Dirac structures characterize not only all Lie algebra structures on $V$, as pointed out by Weinstein [36, but also all Lie algebra structures on subspaces of $V$.

Example 4.2. Given a skew-symmetric bundle map $\widehat{\lambda}: \mathfrak{D} E \rightarrow \mathfrak{J} E$, its graph

$$
L^{\widehat{\lambda}}=\{\mathfrak{d}+\widehat{\lambda}(\mathfrak{d}) \mid \forall \mathfrak{d} \in \mathfrak{D} E\} \subset \mathcal{E}
$$

is clearly a maximal isotropic subbundle. Furthermore, we have $\widehat{\lambda}(\mathfrak{g l}(E)) \subset$ $\operatorname{Hom}(T M, E)$, i.e. $\mathrm{p} \widehat{\lambda}(\Phi)=0$. In fact, $\forall \Phi \in \mathfrak{g l}(E)$, we have

$$
\left\langle\widehat{\lambda}(\Phi), \mathbf{1}_{E}\right\rangle_{E}=\mathbb{p} \widehat{\lambda}(\Phi), \quad\left\langle\widehat{\lambda}\left(\mathbf{1}_{E}\right), \Phi\right\rangle_{E}=\Phi \circ \mathbb{p} \widehat{\lambda}\left(\mathbf{1}_{E}\right) .
$$

Since $\widehat{\lambda}$ is skew-symmetric, we have $\mathrm{p} \widehat{\lambda}(\Phi)=-\Phi \circ \mathrm{p} \widehat{\lambda}\left(\mathbf{1}_{E}\right)$. If we take $\Phi=\mathbf{1}_{E}$, then $\mathrm{p} \widehat{\lambda}\left(\mathbf{1}_{E}\right)=0$. Thus, $\mathrm{p} \widehat{\lambda}(\Phi)=0$.

Let $\lambda: T M \longrightarrow E$ be the induced bundle map of $\hat{\lambda}$. Then we have the following commutative diagram:

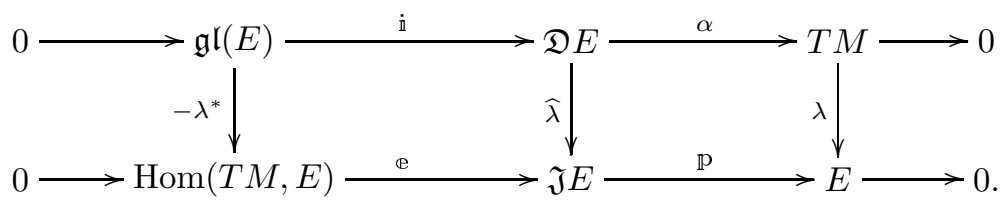

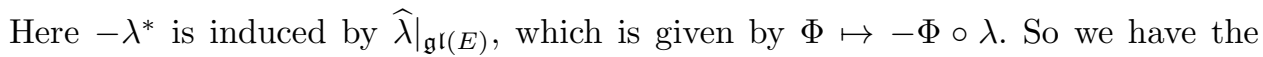
following exact sequence:

$$
0 \longrightarrow \mathbf{G}_{-\lambda^{*}} \longrightarrow L^{\widehat{\lambda}} \longrightarrow \mathbf{G}_{\lambda} \longrightarrow 0
$$


where $\mathbf{G}_{\lambda}=\mathbf{b}\left(L^{\widehat{\lambda}}\right)$ is the graph of $\lambda$ and $\mathbf{G}_{-\lambda^{*}}=L^{\widehat{\lambda}} \cap \operatorname{Hom}(\mathcal{T}, E)$ is the graph of $-\lambda^{*}$.

We claim that the following three statements are equivalent.

1) $L^{\widehat{\lambda}}$ is a Dirac structure.

2) $\mathbb{d} \widehat{\lambda}=0$, regarding $\widehat{\lambda}$ as a map $\mathfrak{D} E \wedge \mathfrak{D} E \rightarrow E$ in the obvious sense:

$$
\widehat{\lambda}(\mathfrak{d}, \mathfrak{r})=\langle\widehat{\lambda}(\mathfrak{d}), \mathfrak{r}\rangle_{E}, \quad \forall \mathfrak{d}, \mathfrak{r} \in \mathfrak{D} E .
$$

3) $\widehat{\lambda}=-\mathbb{d}(\lambda \circ \alpha)$.

In fact, 1) $\Longleftrightarrow 2$ ) is merely some calculations. 3$) \Longrightarrow 2$ ) is trivial. To see the reverse, notice that $\forall \mathfrak{r}, \mathfrak{s} \in \Gamma(\mathfrak{D} E)$,

$$
\begin{aligned}
\mathbb{d} \widehat{\lambda}\left(\mathbf{1}_{E}, \mathfrak{r}, \mathfrak{s}\right) & =\langle\hat{\lambda}(\mathfrak{r}), \mathfrak{s}\rangle_{E}-\mathfrak{r}\left\langle\widehat{\lambda}\left(\mathbf{1}_{E}\right), \mathfrak{s}\right\rangle_{E}+\mathfrak{s}\left\langle\widehat{\lambda}\left(\mathbf{1}_{E}\right), \mathfrak{r}\right\rangle_{E}-\left\langle\hat{\lambda}[\mathfrak{r}, \mathfrak{s}]_{\mathfrak{D}}, \mathbf{1}_{E}\right\rangle \\
& =\hat{\lambda}(\mathfrak{r}, \mathfrak{s})+\mathfrak{r}(\lambda \circ \alpha(\mathfrak{s}))-\mathfrak{s}(\lambda \circ \alpha(\mathfrak{r}))-(\lambda \circ \alpha)[\mathfrak{r}, \mathfrak{s}]_{\mathfrak{D}},
\end{aligned}
$$

which implies that 2) $\Longrightarrow 3$ ).

Thus, any Dirac structure of the type $L^{\widehat{\lambda}}$ is a reducible Dirac structure and totally determined by

$$
\mathbf{b}\left(L^{\widehat{\lambda}}\right)=\mathbf{G}_{\lambda} \subset \mathcal{T},
$$

which is isomorphic to $T M$ and equipped with the induced Lie algebroid structure.

Example 4.3. (See [7) For a skew-symmetric bundle map $\pi: \mathfrak{J} E \rightarrow \mathfrak{D} E$, its graph

$$
L_{\pi}=\{\pi(\mu)+\mu \mid \mu \in \mathfrak{J} E\} \subset \mathcal{E}
$$

is clearly a maximal isotropic subbundle of $\mathcal{E}$. It can be proved that $L_{\pi}$ is a Dirac structure if and only if the following equation holds for all $\mu, \nu \in \Gamma(\mathfrak{J} E)$,

$$
\pi[\mu, \nu]_{\pi}=[\pi(\mu), \pi(\nu)]_{\mathfrak{D}},
$$

where the $\pi$-bracket $[\cdot, \cdot]_{\pi}$ on $\Gamma(\mathfrak{J} E)$ is given by:

$$
[\mu, \nu]_{\pi} \triangleq \mathfrak{L}_{\pi(\mu)} \nu-\mathfrak{L}_{\pi(\nu)} \mu-\mathbb{d} \circ \pi(\mu \wedge \nu) .
$$

To see what $\pi$ encodes, we need to consider the following two situations:

- $\operatorname{rank}(E) \geq 2$. In this case, in [7, we proved that such Dirac structures are in one-to-one correspondence with Lie algebroid structures on $E$. Let us see how Theorem 3.7 recovers this result. On one hand, there is an obvious one-to-one correspondence between Lie algebroid structures $\left(E,[\cdot, \cdot]_{E}, \rho_{E}\right)$ and projective Lie algebroids $\mathbf{G}_{\rho_{E}}$ which are the graphs of $\rho_{E}: E \rightarrow T M$. On the other hand, by Lemma 3.1, any Dirac structure is reducible. Especially, for any Dirac structure $L_{\pi} \subset \mathcal{E}, \mathbf{b}\left(L_{\pi}\right)$ should be a projective Lie algebroid. However, $\mathbf{b}\left(L_{\pi}\right)$ is also a graph and hence there is an induced Lie algebroid structure on $E$. So we conclude that Lie 
algebroid structures on $E$ are in one-to-one correspondence with Dirac structures of the type $L_{\pi}$.

- $\operatorname{rank}(E)=1$. For any reducible Dirac structure $L_{\pi} \subset \mathcal{E}, \mathbf{b}\left(L_{\pi}\right)$ is a projective Lie algebroid. But in general, it may not be a graph and so there is no induced Lie algebroid structure on $E$. However, there is always a local Lie algebra structure on $E$ associated with the Dirac structure $L_{\pi}$ (not necessarily reducible) as proved in 7.

Example 4.4. Consider the case that $A \subset \mathcal{T}$ is an arbitrary line bundle, which is naturally a projective Lie algebroid. In fact, for any neighborhood $\mathcal{U} \subset M$ such that $\left.A\right|_{\mathcal{U}}$ is trivial, i.e. there is a nowhere singular section $a=x+u$, the Lie bracket of $\Gamma\left(\left.A\right|_{\mathcal{U}}\right)$ is given by:

$$
[f a, g a]_{A}=(f x(g)-g x(f)) a, \quad \forall f, g \in C^{\infty}(\mathcal{U}) .
$$

It is easy to check that this bracket is well defined.

The lifted Dirac structure declared by Theorem 3.7 can be constructed by Equation (3.9). Just take any connection $\gamma$. Since $A$ is a line bundle, we have $\Omega_{\gamma}(A) \subset A^{0}$. The lifted Dirac structure is given by $L^{A}=L_{s_{\gamma}, A}=\gamma(A) \oplus A^{0}$.

Example 4.5. Assume that $F \subset E$ is a vector subbundle and $\left(F,[\cdot, \cdot], \rho_{F}\right)$ is a Lie algebroid. Then $\mathbf{G}_{\rho_{F}}$, the graph of $\rho_{F}$ is a projective Lie algebroid. Now we construct the lifted Dirac structure. Evidently, we have

$$
\mathbf{G}_{\rho_{F}}^{0}=\left\{\Phi+\mathfrak{y} \in \operatorname{Hom}(\mathcal{T}, E)\left|\left(\mathfrak{y} \circ \rho_{F}+\Phi\right)\right|_{F}=0\right\} .
$$

Let $L_{1} \subset \mathcal{E}$ be the subset generated by elements of the form $\mathfrak{d}_{m}^{v}+[v]_{m}$, where $m \in M, v \in \Gamma(F), \mathfrak{d}_{m}^{v} \in(\mathfrak{D} E)_{m}$ and they satisfy

$$
\mathfrak{d}_{m}^{v}(u)=\left([v, u]_{F}\right)_{m}, \quad \forall u \in \Gamma(F) .
$$

Let $L=L_{1}+\mathbf{G}_{\rho_{F}}^{0}$. Accordingly, we get an exact sequence:

$$
0 \longrightarrow \mathbf{G}_{\rho_{F}}^{0} \longrightarrow L \longrightarrow \mathbf{b}_{\rho_{F}} \longrightarrow 0 .
$$

It is clear that $L$ is maximal isotropic. Moreover, for all $u, v \in \Gamma(F)$, we have

$$
\left[\mathfrak{d}^{u}, \mathfrak{d}^{v}\right]_{\mathfrak{D} E}=\mathfrak{d}^{[u, v]_{F}}
$$

and it follows that $\Gamma(L)$ is closed under the bracket $\{\cdot, \cdot\}$. Hence $L$ is the lifted Dirac structure.

Example 4.6. We consider a projective Lie algebroid $A$ which is transitive, i.e. $\rho_{A}(A)=\operatorname{pr}_{T M}(A)=T M$. One can construct a map $\vartheta: T M \rightarrow E$ such that $A=\mathbf{G}_{\vartheta} \oplus E_{0}$, where $\mathbf{G}_{\vartheta}$ is the graph of $\vartheta$ and $E_{0}$ is a subbundle of $E$. In this case, $E_{0}$ must be a Lie algebra bundle and we denote its Lie bracket by $[\cdot, \cdot]^{0}$.

For any vector field $x \in \mathfrak{X}(M)$, write $\bar{x}=x+\vartheta(x) \in \Gamma(A)$. There is a suitable connection $\gamma: T M \rightarrow \mathfrak{D} E_{0}$ such that

$$
[\bar{x}, u]_{A}=\gamma(x) u, \quad \forall x \in \mathfrak{X}(M), u \in \Gamma\left(E_{0}\right) .
$$


Define $R: \wedge^{2} T M \rightarrow E_{0}$ by

$$
R(x, y)=[\bar{x}, \bar{y}]_{A}-\overline{[x, y]}, \quad \forall x, y \in \mathfrak{X}(M) .
$$

So the Lie bracket of $\Gamma(A)$ can be written as

$[\bar{x}+u, \bar{y}+v]_{A}=\overline{[x, y]}+R(x, y)+\gamma(x) v-\gamma(y) u+[u, v]^{0}, \quad \forall \bar{x}+u, \bar{y}+v \in \Gamma(A)$.

Under the structure defined by the given data $(\gamma, R), A=\mathbf{G}_{\vartheta} \oplus E_{0}$ is a Lie algebroid if and only if $\forall x, y, z \in \mathfrak{X}(M), u, v \in \Gamma\left(E_{0}\right)$, the following compatibility conditions hold

$$
\begin{aligned}
{[\gamma(x) u, v]^{0}+[u, \gamma(x) v]^{0} } & =\gamma(x)[u, v]^{0}, \\
{[\gamma(x), \gamma(y)]_{\mathfrak{D}}-\gamma[x, y] } & =\operatorname{ad}_{R(x, y)}^{E_{0}}, \\
R([x, y], z)-\gamma(x) R(y, z)+\text { c.p. } & =0 .
\end{aligned}
$$

We extend the connection $\gamma$ in the vector bundle $E_{0}$ to a connection $\widetilde{\gamma}$ in the vector bundle $E$. By (3.7), we have

$$
\Omega_{\widetilde{\gamma}}(\bar{x}+u, \bar{y}+v)=R(x, y)+[u, v]^{0}-\left(d^{\widetilde{\gamma}} \vartheta\right)(x, y),
$$

where

$$
\left(d^{\widetilde{\gamma}} \vartheta\right)(x, y)=\widetilde{\gamma}(x) \vartheta(y)-\widetilde{\gamma}(y) \vartheta(x)-\vartheta[x, y] .
$$

The lifted Dirac structure, given by Theorem 3.7 can be expressed by (3.9).

In particular, if $A=\mathcal{T}=T M \oplus E$ (so that we may take $\vartheta=0$ ), then

$$
L_{s_{\gamma}, \mathcal{T}}=\left\{\gamma(x+u)+i_{x} R+\operatorname{ad}_{u}^{E} \mid \forall x+u \in T M \oplus E\right\} .
$$

We note that the above construction of projective Lie algebroids includes a standard type of Lie algebroids, known as a semi-direct-sum. If $E$ is a vector bundle over $M$ which admits a flat connection $\nabla: T M \rightarrow \mathfrak{D} E$, then the direct sum $T M \oplus E$ has a Lie algebroid structure over $M$, for which the anchor is the projection to $T M$ and the Lie bracket is given by:

$$
[x+u, y+v] \triangleq[x, y]+\nabla_{x} v-\nabla_{y} u, \quad \forall x+u, y+v \in \Gamma(T M \oplus E) .
$$

\section{The Normalizer of Dirac Structures}

In this and the next section, we always assume that Lie algebroids under consideration are not zero. For a Lie algebroid $A$, call $\operatorname{Der}(A)$, the set of Lie derivations of $A$ :

$$
\operatorname{Der}(A)=\left\{\delta \in \Gamma(\mathfrak{D} A) \mid \delta\left[a_{1}, a_{2}\right]_{A}=\left[\delta a_{1}, a_{2}\right]_{A}+\left[a_{1}, \delta a_{2}\right]_{A}, \quad \forall a_{1}, a_{2} \in \Gamma(A),\right\}
$$

the derivation algebra of $A$.

Definition 5.1. The normalizer $N_{C}$ of a subbundle $C$ of the omni-Lie algebroid $\mathcal{E}=\mathfrak{D} E \oplus \mathfrak{J} E$ is composed of all the sections of $\mathcal{E}$ that preserve $\Gamma(C)$ from the left side, that is,

$$
N_{C}=\{X \in \Gamma(\mathcal{E}) \mid\{X, Y\} \in \Gamma(C), \quad \forall Y \in \Gamma(C)\}
$$


It is easy to see that the normalizer $N_{C}$ of $C$ is a Leibniz subalgebrab of $\Gamma(\mathcal{E})$. For any $X \in \Gamma(\mathcal{E})$, we introduce the falling operator

$$
(\cdot) \text { : } \quad \Gamma(\mathcal{E}) \longrightarrow \Gamma(\mathfrak{D T}),
$$

which is defined by

$$
X_{\bullet}(t) \triangleq \mathbf{b}\{X, Y\}, \quad \forall t \in \Gamma(\mathcal{T}),
$$

where $Y \in \Gamma(\mathcal{E})$ satisfying $\mathbf{b}(Y)=t$. By Lemma 2.6, this is well defined and if $h \in \Gamma(\operatorname{Hom}(\mathcal{T}, E)), h_{\bullet}=h$.

In this section, we study the normalizer $N_{L}$ of a Dirac structure $L$. Using the falling operator defined above, we establish the relation between the normalizer $N_{L}$ of a reducible Dirac structure $L$ and the derivation algebra $\operatorname{Der}(\mathbf{b}(L))$ of the projective Lie algebroid $\mathbf{b}(L)$.

Proposition 5.2. The falling operator $(\cdot)$. is a morphism of Leibniz algebras. Furthermore, $\forall X \in \Gamma(\mathcal{E}), t \in \Gamma(\mathcal{T})$, we have

$$
\operatorname{pr}_{T M}\left(X_{\bullet}(t)\right)=\left[\alpha \circ \rho(X), p r_{T M}(t)\right]=\left[\alpha\left(X_{\bullet}\right), p r_{T M}(t)\right] .
$$

Conversely, given any $\delta \in \Gamma(\mathfrak{D T})$ satisfying Equation (5.3), there exists an $X_{\delta} \in$ $\Gamma(\mathcal{E})$ such that $X_{\delta \bullet}=\delta$.

Proof. For all $X, Y \in \Gamma(\mathcal{E}), t \in \Gamma(\mathcal{T})$ and $Z \in \Gamma(\mathcal{E})$ satisfying $\mathbf{b}(Z)=t$, we have

$$
\begin{aligned}
\{X, Y\}_{\bullet}(t) & =\mathbf{b}\{\{X, Y\}, Z\}=\mathbf{b}(\{X,\{Y, Z\}\}-\{Y,\{X, Z\}\} \\
& =X \bullet \mathbf{b}\{Y, Z\}-Y_{\bullet} \mathbf{b}\{X, Z\} \\
& =X \bullet \circ Y \bullet(t)-Y_{\bullet} \circ X_{\bullet}(t) \\
& =\left[X_{\bullet}, Y_{\bullet}\right]_{\mathfrak{D}}(t),
\end{aligned}
$$

which implies that the falling operator $(\cdot)$. is a morphism of Leibniz algebras.

Given $X=\mathfrak{d}+\mu$ and $Z=\mathfrak{r}+\nu$ such that $\operatorname{pr}_{T M}(t)=\alpha(\mathfrak{r})$, we have

$$
\begin{aligned}
\operatorname{pr}_{T M}\left(X_{\bullet}(t)\right) & =\operatorname{pr}_{T M} \mathbf{b}\{X, Z\} \\
& =[\alpha(\mathfrak{d}), \alpha(\mathfrak{r})]=\left[\alpha \circ \rho(X), p r_{T M}(t)\right] \\
& =\left[\alpha(X \bullet), p r_{T M}(t)\right],
\end{aligned}
$$

which implies Equation (5.3).

Suppose that $\delta \in \Gamma(\mathfrak{D T})$ satisfies Equation (5.3). Write $x=\alpha(\delta)$ and define $\chi=p r_{E} \circ \delta$. One has

$$
\chi(f t)=x(f) p r_{E}(t)+f \chi(t), \quad \forall f \in C^{\infty}(M) .
$$

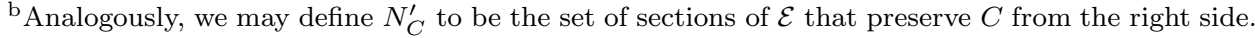
But it is not a Leibniz subalgebra.
} 
Therefore, $\left.\chi\right|_{\mathfrak{X}(M)}$ is $C^{\infty}(M)$-linear and there is an associated $X_{M} \in$ $\Gamma(\operatorname{Hom}(T M, E))$. Moreover, $\left.\chi\right|_{\Gamma(E)}$ is a derivation and there is an associated $X_{E} \in \Gamma(\mathfrak{D} E)$ such that $\alpha\left(X_{E}\right)=x$. In turn, the operation of $\delta$ can be expressed as

$$
\delta(y+v)=[x, y]+X_{E}(v)+X_{M}(y), \quad \forall y+v \in \Gamma(\mathcal{T}) .
$$

Let $X_{\delta}=X_{E}+X_{M} \in \Gamma(\mathfrak{D} E) \oplus \Gamma(\operatorname{Hom}(T M, E)) \subset \Gamma(\mathcal{E})$. We claim that $X_{\delta}=\delta$. In fact, for any $y+v \in \Gamma(\mathcal{T})=\Gamma(T M) \oplus \Gamma(E)$ and $Y=\mathfrak{r}+\nu \in \Gamma(\mathcal{E})$ satisfying $\alpha(\mathfrak{r})=y$ and $\mathbb{p}(\nu)=v$, we have

$$
\begin{aligned}
X_{\delta \bullet}(y+v) & =\mathbf{b}\{X, Y\}=\mathbf{b}\left(\left[X_{E}, \mathfrak{r}\right]_{\mathfrak{D}}+\mathfrak{L}_{X_{E}} \nu-\mathfrak{L}_{\mathfrak{r}} X_{M}+\mathbb{d}\left(X_{M}(y)\right)\right) \\
& =[x, y]+X_{E}(v)+X_{M}(y)=\delta(y+v) .
\end{aligned}
$$

Let $A \subset \mathcal{T}$ be a projective Lie algebroid and $\operatorname{Inn}(A)$ the set of inner derivations, which consists of operators $[a, \cdot]_{A}$, where $a \in \Gamma(A)$. Denote the set of external derivations by $\operatorname{Ext}(A)$, i.e.

$$
\operatorname{Ext}(A)=\operatorname{Der}(A) / \operatorname{Inn}(A) .
$$

By Theorem 3.7 there is a unique lifted Dirac structure $L^{A}$ such that $A$ is the quotient Lie algebroid of $L^{A}$. Concerning the relation between the normalizer $N_{L^{A}}$ and the derivation algebra $\operatorname{Der}(A)$, we have

Theorem 5.3. If $X \in N_{L^{A}}$, then $\left.X_{\bullet}\right|_{A} \in \operatorname{Der}(A)$. Conversely, for any $\delta \in \operatorname{Der}(A)$, there exists an $X_{\delta} \in N_{L^{A}}$, such that $\left(X_{\delta}\right) .\left.\right|_{A}=\delta$. Moreover, we have the following commutative diagram where the two rows are exact sequences:

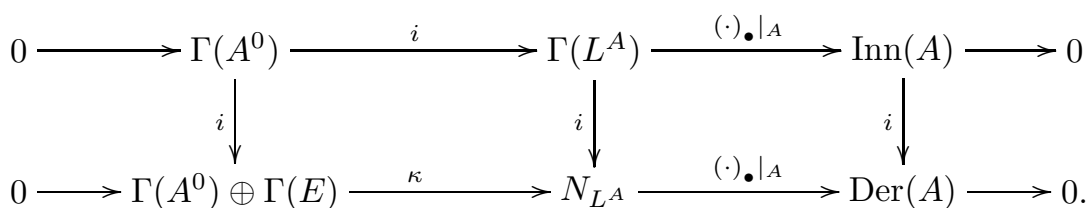

Here $i$ is the inclusion. The map $\kappa$ is defined by $\kappa(\phi+u)=\phi+\mathbb{d} u, \forall \phi \in \Gamma\left(A^{0}\right), u \in$ $\Gamma(E)$. In particular, $X_{\bullet} \mid{ }_{A} \in \operatorname{Inn} A$ if and only if $X=l+\mathbb{d} u$, for some $l \in \Gamma\left(L^{A}\right)$, $u \in \Gamma(E)$.

Proof. If $X \in N_{L^{A}}$, then for any $a_{1}, a_{2} \in \Gamma(A)$, we can find $l_{1}, l_{2} \in \Gamma\left(L^{A}\right)$ such that $\mathbf{b}\left(l_{i}\right)=a_{i}$. Hence

$$
\begin{aligned}
X_{\bullet}\left[a_{1}, a_{2}\right]_{A} & =\mathbf{b}\left\{X,\left\{l_{1}, l_{2}\right\}\right\}=\mathbf{b}\left\{\left\{X, l_{1}\right\}, l_{2}\right\}+\mathbf{b}\left\{l_{1},\left\{X, l_{2}\right\}\right\} \\
& =\left[\mathbf{b}\left\{X, l_{1}\right\}, a_{2}\right]_{A}+\left[a_{1}, \mathbf{b}\left\{X, l_{2}\right\}\right]_{A} \\
& =\left[X \bullet a_{1}, a_{2}\right]_{A}+\left[a_{1}, X_{\bullet} a_{2}\right]_{A},
\end{aligned}
$$

which implies that $X_{\bullet} \mid{ }_{A} \in \operatorname{Der}(A)$.

Conversely, given any $\delta \in \operatorname{Der}(A)$, set $x=\alpha(\delta) \in \mathfrak{X}(M)$ and find an extension $\widetilde{\delta} \in \operatorname{Der}(\mathcal{T})$ of $\delta$, that is, $\alpha(\widetilde{\delta})=x$ and $\left.\widetilde{\delta}\right|_{\Gamma(A)}=\delta$. Since the elements of $\operatorname{Der}(A)$ 
satisfy (5.3) and by Proposition 5.2 there is an $X_{\widetilde{\delta}}=X_{E}+X_{M}$ such that $X_{\widetilde{\delta}}=\widetilde{\delta}$, i.e. $X_{\widetilde{\delta}}||_{A}=\delta$.

Next we prove $X_{\widetilde{\delta}} \in N_{L^{A}}$. For all $l \in \Gamma\left(L^{A}\right)$, it is evident that $\left\{X_{\widetilde{\delta}}, l\right\} \in$ $\Gamma\left(\mathbf{b}^{-1}(A)\right)$. Furthermore, $\forall Y \in \Gamma\left(\mathbf{b}^{-1}(A)\right)$, we have

$$
\begin{aligned}
\mathbf{b}\left\{\left\{X_{\widetilde{\delta}}, l\right\}, Y\right\} & =\mathbf{b}\left\{X_{\widetilde{\delta}},\{l, Y\}\right\}-\mathbf{b}\left\{l,\left\{X_{\widetilde{\delta}, Y}\right\}\right\} \\
& =X_{\widetilde{\delta}}[\mathbf{b} l, \mathbf{b} Y]_{A}-\left[\mathbf{b} l, X_{\widetilde{\delta}}(\mathbf{b} Y)\right]_{A} \\
& =\left[X_{\widetilde{\delta}}(\mathbf{b} l), \mathbf{b}(Y)\right]_{A}=\left[\mathbf{b}\left\{X_{\widetilde{\delta}}, l\right\}, \mathbf{b} Y\right]_{A},
\end{aligned}
$$

which implies that $X_{\widetilde{\delta}} \in N_{L^{A}}$.

For an $X \in N_{L^{A}}$ satisfying $X_{\bullet}(\Gamma(A))=0$, it is easy to see that $\alpha\left(X_{\bullet}\right)=0$, i.e. $\alpha \circ \rho(X)=0$. So we are able to write

$$
X=\Phi+\mathfrak{y}+\mathbb{d} u, \quad \text { where } \Phi \in \Gamma(\mathfrak{g l}(E)), \mathfrak{y} \in \Gamma(\operatorname{Hom}(T M, E)), u \in \Gamma(E) .
$$

Clearly, $\{\mathbb{d} u, \cdot\}=0$. By Lemma 2.6, we have $\Phi+\mathfrak{y} \in \Gamma\left(A^{0}\right)$, which implies that $\operatorname{ker}\left(\left.X \bullet\right|_{A}\right)=\Gamma\left(A^{0}\right) \oplus \Gamma(E)$. The remaining statements of the theorem are easy to be checked and we omit the details.

Example 5.4. For a reducible Dirac structure $L_{\pi}$ given in Example 4.3, we consider its normalizer. For $u \in \Gamma(E)$, since we have $\left\{\mathbb{d} u, L_{\pi}\right\}=0$, it suffices to consider elements of the form $\mathfrak{d}+\mathfrak{y} \in \Gamma(\mathcal{E})$, where $\mathfrak{y} \in \Gamma(\operatorname{Hom}(T M, E))$. Rewrite $\mathfrak{d}+\mathfrak{y}=$ $\mathfrak{d}-\pi(\mathfrak{y})+\pi(\mathfrak{y})+\mathfrak{y}$ where $\pi(\mathfrak{y})+\mathfrak{y} \in L_{\pi}$. We have

$\mathfrak{d}+\mathfrak{y} \in N_{L_{\pi}} \Longleftrightarrow \mathfrak{d}-\pi(\mathfrak{y}) \in N_{L_{\pi}} \Longleftrightarrow \mathfrak{L}_{\mathfrak{d}-\pi(\mathfrak{y})} \circ \pi=\pi \circ \mathfrak{L}_{\mathfrak{d}-\pi(\mathfrak{y})} \Longleftrightarrow d(\mathfrak{d}-\pi(\mathfrak{y}))=0$.

Here the coboundary operator $d$ is associated with cochain complex $\Gamma\left(\operatorname{Hom}\left(\wedge^{\bullet} \mathfrak{J} E, E\right)\right)$ and the representation $\pi: \mathfrak{J} E \longrightarrow \mathfrak{D} E$, which is known as the adjoint representation 9|29. From this we get the following exact sequence:

$$
0 \rightarrow \Gamma(\operatorname{Hom}(T M, E)) \oplus \Gamma(E) \stackrel{\kappa}{\longrightarrow} N_{L_{\pi}} \stackrel{p}{\longrightarrow} B(\mathfrak{J} E, E) \cap \mathfrak{D} E \rightarrow 0,
$$

where $B(\mathfrak{J} E, E)$ is the set of 1 -cocycles and the maps $\kappa, p$ are given by

$$
\kappa(\mathfrak{y}+u)=\pi(\mathfrak{y})+\mathfrak{y}+\mathbb{d} u, \quad p(\mathfrak{d}+\mathfrak{y}+[u])=\mathfrak{d}-\pi(\mathfrak{y}),
$$

where $\mathfrak{y} \in \Gamma(\operatorname{Hom}(T M, E)), \mathfrak{d} \in \Gamma(\mathfrak{D} E), u \in \Gamma(E)$.

\section{Cohomology of Dirac Structures}

By Proposition 2.5 for any Dirac structure $L \subset \mathcal{E}$, there is a representation $\rho_{L}$ on $E$. Let $d_{L}: \Gamma\left(\operatorname{Hom}\left(\wedge^{\bullet} L, E\right)\right) \rightarrow \Gamma\left(\operatorname{Hom}\left(\wedge^{\bullet+1} L, E\right)\right)$ be the associated coboundary operator. In this section, we study the cohomology group $\mathrm{H}^{\bullet}\left(L, \rho_{L}\right)$ and explore the relation between $N_{L}$ and $\mathrm{H}^{1}\left(L, \rho_{L}\right)$. We also study the deformation of a Dirac structure, which is related with $\mathrm{H}^{2}\left(L, \rho_{L}\right)$.

Let $L \subset \mathcal{E}$ be a Dirac structure. For any $X \in \Gamma(\mathcal{E}), \omega_{X}=(X, \cdot)_{E}: L \longrightarrow E$ naturally defines a 1-cochain. We first prove the following fact.

Proposition 6.1. $X \in N_{L} \Longleftrightarrow d_{L} \omega_{X}=0$. 
Proof. For any $l_{1}, l_{2} \in \Gamma(L)$, we have

$$
\begin{aligned}
d_{L} \omega_{X}\left(l_{1}, l_{2}\right)= & \rho_{L}\left(l_{1}\right) \omega_{X}\left(l_{2}\right)-\rho_{L}\left(l_{2}\right) \omega_{X}\left(l_{1}\right)-\omega_{X}\left(\left\{l_{1}, l_{2}\right\}\right) \\
= & \rho_{L}\left(l_{1}\right)\left(X, l_{2}\right)_{E}-\rho_{L}\left(l_{2}\right)\left(X, l_{1}\right)_{E}-\left(X,\left\{l_{1}, l_{2}\right\}\right)_{E} \\
= & \left(\left\{l_{1}, X\right\}, l_{2}\right)_{E}-\left(\left\{l_{2}, X\right\}, l_{1}\right)_{E}-\left(X,\left\{l_{2}, l_{1}\right\}\right)_{E} \\
= & -\left(\left\{X, l_{1}\right\}, l_{2}\right)_{E}+\left(\left\{X, l_{2}\right\}, l_{1}\right)_{E}-\left(X,\left\{l_{2}, l_{1}\right\}\right)_{E} \\
& +\left(2 \mathbb{d}\left(l_{1}, X\right)_{E}, l_{2}\right)_{E}-\left(2 \mathbb{d}\left(l_{2}, X\right)_{E}, l_{1}\right)_{E} \\
= & -2\left(\left\{X, l_{1}\right\}, l_{2}\right)_{E}-\left(X,\left\{l_{2}, l_{1}\right\}\right)_{E}-\rho_{L}\left(l_{1}\right)\left(X, l_{2}\right)_{E}+\rho_{L}\left(l_{2}\right)\left(X, l_{1}\right)_{E} \\
= & -2\left(\left\{X, l_{1}\right\}, l_{2}\right)_{E}-d_{L} \omega_{X}\left(l_{1}, l_{2}\right) .
\end{aligned}
$$

Therefore,

$$
d_{L} \omega_{X}\left(l_{1}, l_{2}\right)=-\left(\left\{X, l_{1}\right\}, l_{2}\right)_{E} .
$$

Since $L^{\perp}=L$, the above equality implies that $X \in N_{L} \Longleftrightarrow d_{L} \omega_{X}=0$.

Proposition 6.2. Let $A \subset \mathcal{T}$ be a projective Lie algebroid and $L^{A}$ the lifted Dirac structure, for any $X \in N_{L^{A}}, \omega_{X}$ is a coboundary if and only if $X_{\bullet} \in \operatorname{Inn} A$

Proof. By definition, $\omega_{X}=d_{L^{A}} u$, for some $u \in \Gamma(E)$, if and only if

$$
\left(X-2 \mathbb{d} u, L^{A}\right)_{E}=0 \Longleftrightarrow X=2 \mathbb{d} u+l, \quad \text { for some } l \in \Gamma\left(L^{A}\right) .
$$

So the conclusion follows directly by Theorem 5.3

Corollary 6.3. With the above notations, there is a natural inclusion

$$
i: \operatorname{Ext}(A) \longrightarrow \mathrm{H}^{1}\left(L^{A}, \rho_{L^{A}}\right), \quad i(\delta)=\omega_{X_{\delta}}, \quad \forall \delta \in \operatorname{Der}(A) .
$$

where $\operatorname{Ext}(A)$ is defined by (5.4) and $X_{\delta}$ is given in Theorem 5.3 .

Proof. By Theorem [5.3, for any $\delta \in \operatorname{Der}(A)$, there is an $X_{\delta} \in N_{L^{A}}$ such that $\left.X_{\delta}\right|_{A}=\delta$. By Proposition 6.1 $i(\delta)=\omega_{X_{\delta}}$ is closed.

To see that $i$ is well defined, we note that $\omega_{X_{\delta}}$ does not depend on the choice of $X_{\delta}$ (by Theorem [5.3). And if $\delta \in \operatorname{Inn}(A)$, then by Theorem[5.3 again, $X_{\delta}=l+2 \mathrm{~d} u$, where $l \in \Gamma\left(L^{A}\right)$ and $u \in \Gamma(E)$. Therefore, $\omega_{X_{\delta}}=d_{L^{A}} u$ is exact.

Finally, the previous proposition implies that $i$ is injective.

Suppose that $E$ and $E^{*}$ are both Lie algebroids, respectively, with anchors $\alpha$ and $\alpha^{*}$. Let $d_{*}: \Gamma\left(\wedge^{\bullet} E\right) \rightarrow \Gamma\left(\wedge^{\bullet+1} E\right)$ be the Lie algebroid coboundary operator associated with the Lie algebroid structure on $E^{*}$. So we have $d_{*}^{2}=0$. By definition, $\left(E, E^{*}\right)$ is a Lie bialgebroid if the following equality holds:

$$
d_{*}[u, v]=\left[d_{*} u, v\right]+\left[u, d_{*} v\right], \quad \forall u, v \in \Gamma(E) .
$$

(For more details about Lie bialgebroids, see 23 and 22]). The operator $d_{*}: \Gamma(E) \rightarrow \Gamma\left(\wedge^{2} E\right)$ can be lifted to a bundle map $\hat{d}_{*}: \mathfrak{J} E \rightarrow \wedge^{2} E$, defined by

$$
\hat{d}_{*}(\mathbb{d} u) \triangleq d_{*} u, \quad \hat{d}_{*}(\mathrm{~d} f \otimes u) \triangleq d_{*} f \wedge u, \quad \forall u \in \Gamma(E), f \in C^{\infty}(M) .
$$


In [7, we proved that a Lie algebroid structure on $E$ can be lifted to a bundle map $\pi: \mathfrak{J} E \rightarrow \mathfrak{D} E$, which is also a representation of the jet Lie algebroid $\left(\mathfrak{J} E,[\cdot, \cdot]_{\pi}, \alpha \circ\right.$ $\pi$ ) on $E$, where $\pi$ is given by $\pi(\mathbb{d} u)(v)=[u, v]$ (known as the adjoint representation of a Lie algebroid) and the Lie bracket $[\cdot, \cdot]_{\pi}$ is given by (4.1). So we have an induced tensor representation $\widetilde{\pi}$ of $\mathfrak{J} E$ on $\wedge^{2} E$ given by

$$
\tilde{\pi}(\mathbb{d} u)(\mathcal{W})=[u, \mathcal{W}], \quad \widetilde{\pi}(\mathrm{d} f \otimes u)(\mathcal{W})=[\mathcal{W}, f] \wedge u, \quad \forall \mathcal{W} \in \Gamma\left(\wedge^{2} E\right) .
$$

\section{Proposition 6.4.}

1) The pair $\left(E, E^{*}\right)$ is a Lie bialgebroid if and only if $\hat{d}_{*}$ is a 1-cocycle.

2) The pair $\left(E, E^{*}\right)$ is a coboundary Lie bialgebroid (i.e. $d_{*}=[\tau, \cdot]$, for some $\left.\tau \in \Gamma\left(\wedge^{2} E\right)\right)$ if and only if $\hat{d}_{*}$ is a coboundary.

Proof. For all $u, v \in \Gamma(E)$ and $f, g \in C^{\infty}(M)$, we have the following three formulas which are given in [7]:

$$
\begin{aligned}
{[\mathrm{d} u, \mathbb{d} v]_{\pi} } & =\mathbb{d}[u, v], \\
{[\mathrm{d} u, \mathrm{~d} f \otimes v]_{\pi} } & =\mathrm{d} \rho(u)(f) \otimes v+\mathrm{d} f \otimes[u, v], \\
{[\mathrm{d} f \otimes u, \mathrm{~d} g \otimes v]_{\pi} } & =\rho(u)(g)(\mathrm{d} f \otimes v)-\rho(v)(f)(\mathrm{d} g \otimes u) .
\end{aligned}
$$

Denote the coboundary operator associated with the representation $\tilde{\pi}$ by $\mathcal{D}$. We have

$$
\begin{aligned}
\mathcal{D}\left(\hat{d}_{*}\right)(\mathbb{d} u, \mathbb{d} v) & =\widetilde{\pi}(\mathbb{d} u) \hat{d}_{*}(\mathbb{d} v)-\widetilde{\pi}(\mathbb{d} v) \hat{d}_{*}(\mathbb{d} u)-\hat{d}_{*}\left([\mathrm{~d} u, \mathbb{d} v]_{\pi}\right) \\
& =\left[u, d_{*} v\right]-\left[v, d_{*} u\right]-d_{*}[u, v], \\
\mathcal{D}\left(\hat{d}_{*}\right)(\mathbb{d} u, \mathrm{~d} f \otimes v) & =\left(d_{*}[u, f]-\left[d_{*} u, f\right]-\left[u, d_{*} f\right]\right) \wedge v, \\
\mathcal{D}\left(\hat{d}_{*}\right)(\mathrm{d} f \otimes u, \mathrm{~d} g \otimes v) & =\left(\left[d_{*} f, g\right]+\left[f, d_{*} g\right]\right) \wedge u \wedge v .
\end{aligned}
$$

which implies that $\left(E, E^{*}\right)$ is a Lie bialgebroid if and only if $\hat{d}_{*}$ is closed.

It is clear that $d_{*}=[\tau, \cdot] \Longleftrightarrow \hat{d}_{*}=\mathcal{D} \tau$, which implies (2).

Finally we consider the deformation of a projective Lie algebroid $A$ and its lifted Dirac structure $L^{A}$. Let $\Omega: A \wedge A \longrightarrow A \cap E$ be a bundle map. Consider an $\varepsilon$-parameterized family of brackets

$$
[a, b]_{A}^{\varepsilon}=[a, b]_{A}+\varepsilon \Omega(a, b), \quad \forall a, b \in \Gamma(A) .
$$

If every $\varepsilon$ - bracket endows $A$ a projective Lie algebroid structure, we say that $\Omega$ generates a deformation of the projective Lie algebroid $A$. Evidently, this requirement is equivalent to the following compatibility conditions:

$$
\begin{aligned}
\Omega\left([a, b]_{A}, c\right)+[\Omega(a, b), c]_{A}+\text { c.p. } & =0 \\
\Omega(\Omega(a, b), c)+\text { c.p. } & =0 .
\end{aligned}
$$


Equation (6.5) means that $\Omega$ itself defines a (fibrewise) Lie bracket. Furthermore, for all $l_{1}, l_{2}, l_{3} \in \Gamma\left(L^{A}\right)$, we have

$$
\begin{aligned}
d_{L^{A}} \mathbf{b}^{*} \Omega\left(l_{1}, l_{2}, l_{3}\right) & =\rho_{L^{A}}\left(l_{1}\right) \mathbf{b}^{*} \Omega\left(l_{2}, l_{3}\right)+c . p .+\mathbf{b}^{*} \Omega\left(\left\{l_{1}, l_{2}\right\}, l_{3}\right)+c . p . \\
& =\rho_{L^{A}}\left(l_{1}\right) \Omega\left(\mathbf{b} l_{2}, \mathbf{b} l_{3}\right)+c . p .+\Omega\left(\mathbf{b}\left\{l_{1}, l_{2}\right\}, \mathbf{b} l_{3}\right)+c . p . \\
& =\mathbf{b}\left\{l_{1}, \mathbb{d} \Omega\left(\mathbf{b} l_{2}, \mathbf{b} l_{3}\right)\right\}+c . p .+\Omega\left(\left[\mathbf{b} l_{1}, \mathbf{b} l_{2}\right]_{A}, \mathbf{b} l_{3}\right)+c . p . \\
& =\left[\mathbf{b} l_{1}, \Omega\left(\mathbf{b} l_{2}, \mathbf{b} l_{3}\right)\right]_{A}+c . p .+\Omega\left(\left[\mathbf{b} l_{1}, \mathbf{b} l_{2}\right]_{A}, \mathbf{b} l_{3}\right)+c . p .
\end{aligned}
$$

which implies that Equation (6.4) is equivalent to the requirement that $\mathbf{b}^{*} \Omega$ is closed.

Since there is a one-to-one correspondence between reducible Dirac structures and projective Lie algebroids, we can associate a deformation of the Dirac structure $L^{A}$ to the deformation of the projective Lie algebroid $A$. Denote the deformed projective Lie algebroid by $A_{\varepsilon}$, then the deformed Dirac structure $L^{A_{\varepsilon}}$ is give by

$$
L^{A_{\varepsilon}}=\left\{l+h \mid l \in L_{A}, h \in \operatorname{Hom}(\mathcal{T}, E) \text {, s.t., } h(a)=\Omega(\mathbf{b}(l), a), \quad \forall a \in A\right\} .
$$

An interesting problem is to consider a deformation $\Omega$ which is a coboundary:

$$
\mathbf{b}^{*} \Omega=d_{L^{A}} \omega_{X}, \quad \text { for some } X \in \Gamma(\mathcal{E}) .
$$

Proposition 6.5. Let $\Omega: A \wedge A \longrightarrow A \cap E$ be a bundle map. If $\mathbf{b}^{*} \Omega=d_{L^{A}} \omega_{X}$ for some $X \in \Gamma(\mathcal{E})$, then $X \in N_{A^{0}}=N_{\mathbf{b}^{-1}(A)}$. Moreover, we have

$$
\Omega\left(a_{1}, a_{2}\right)=\frac{1}{2}\left(\left[X \bullet a_{1}, a_{2}\right]_{A}+\left[a_{1}, X_{\bullet} a_{2}\right]_{A}-X_{\bullet}\left[a_{1}, a_{2}\right]_{A}\right), \quad \forall a_{1}, a_{2} \in \Gamma(A) .
$$

Furthermore, $\Omega$ generates a deformation of the projective Lie algebroid $A$ if and only if

$$
\left[T^{X}(a, b), c\right]_{A}+T^{X}\left([a, b]_{A}, c\right)+c . p .=0, \quad \forall a, b, c \in \Gamma(A),
$$

where $T^{X}: \Gamma(A) \wedge \Gamma(A) \rightarrow \Gamma(A)$ is defined by

$$
T^{X}(a, b) \triangleq X_{\bullet}\left(\left[X_{\bullet} a, b\right]_{A}+\left[a, X_{\bullet} b\right]_{A}-X_{\bullet}[a, b]_{A}\right)-\left[X \bullet a, X_{\bullet} b\right]_{A} .
$$

Conversely, for any $X \in N_{\mathbf{b}^{-1}(A)}=N_{A^{0}}$ satisfying (6.8), $\Omega$ defined by equation (6.7) is a bundle map from $A \wedge A$ to $A \cap E$ that generates a deformation of $A$ and relation (6.6) holds.

Proof. By Equations (6.1) and (6.6), for all $\theta \in \Gamma\left(A^{0}\right), l \in \Gamma\left(L^{A}\right)$, we have

$$
0=\left(\mathbf{b}^{*} \Omega\right)(\theta, l)=d_{L^{A}} \omega_{X}(\theta, l)=-(\{X, \theta\}, l)_{E} .
$$

Thus, $\{X, \theta\} \in \Gamma\left(L^{A} \cap \operatorname{Hom}(\mathcal{T}, E)\right)=\Gamma\left(A^{0}\right)$, i.e. $X \in N_{A^{0}}$. For any $Y \in \Gamma\left(\mathbf{b}^{-1}(A)\right)$, we have

$$
\begin{aligned}
\{X, \theta\}(\mathbf{b} Y) & =2(\{X, \theta\}, Y)_{E}=2 \rho(X)(\theta, Y)_{E}-2(\theta,\{X, Y\})_{E} \\
& =\rho(X) \theta(\mathbf{b} Y)-\theta \circ \mathbf{b}\{X, Y\}
\end{aligned}
$$


which implies that $\theta \circ \mathbf{b}\{X, Y\}=0$, i.e. $X \in N_{\mathbf{b}^{-1}(A)}$.

Let $l_{i} \in \Gamma\left(L^{A}\right)$ and $\mathbf{b}\left(l_{i}\right)=a_{i}$. By some straightforward computation, we have

$$
\begin{aligned}
\Omega\left(a_{1}, a_{2}\right) & =\left(\mathbf{b}^{*} \Omega\right)\left(l_{1}, l_{2}\right)=d_{L^{A}} \omega_{X}\left(l_{1}, l_{2}\right) \\
& =-\left(\left\{X, l_{1}\right\}, l_{2}\right)_{E}=-\frac{1}{2} \mathbf{b}\left(\left\{\left\{X, l_{1}\right\}, l_{2}\right\}+\left\{l_{2},\left\{X, l_{1}\right\}\right\}\right) \\
& =-\frac{1}{2} \mathbf{b}\left(\left\{X,\left\{l_{1}, l_{2}\right\}\right\}-\left\{l_{1},\left\{X, l_{2}\right\}\right\}+\left\{l_{2},\left\{X, l_{1}\right\}\right\}\right) \\
& =\frac{1}{2}\left(\left[X \bullet a_{1}, a_{2}\right]_{A}+\left[a_{1}, X \bullet a_{2}\right]_{A}-X \bullet\left[a_{1}, a_{2}\right]_{A}\right),
\end{aligned}
$$

which implies Equation (6.7).

If $\Omega$ generates a deformation of the projective Lie algebroid $A, \Omega$ itself defines a fibrewise Lie bracket. It is easy to see that this is equivalent to (6.8). The other conclusions can be easily checked.

For a Lie algebroid $(\mathcal{A},[\cdot, \cdot], \alpha)$, a Nijenhuis operator is a bundle map $N: \mathcal{A} \rightarrow$ $\mathcal{A}$ such that the following equality holds

$$
T^{N}(a, b) \triangleq N([N a, b]+[a, N b]-N[a, b])-[N a, N b]=0, \quad \forall a, b \in \Gamma(\mathcal{A}) .
$$

It induces a new Lie algebroid $\left(\mathcal{A},[\cdot, \cdot]_{N}, \alpha_{N}\right)$, where $\alpha_{N}=\alpha \circ N$ and

$$
[a, b]_{N}=[N a, b]+[a, N b]-N[a, b] .
$$

In fact, $T^{N}=0$ is only a sufficient condition for the bracket operation $[\cdot, \cdot]_{N}$ being a Lie bracket. The necessary and sufficient condition is

$$
\left[a, T^{N}(b, c)\right]+T^{N}(a,[b, c])+c . p .=0 .
$$

The role of the operator $X_{\bullet}: \Gamma(A) \rightarrow \Gamma(A)$ is just like that of a Nijenhuis operator. In general, $X_{\bullet}$ is not a bundle map, but it still induces a twist of the Lie algebroid. In fact, $X_{\bullet}$ is a bundle map if and only if $X \in \Gamma(\operatorname{Hom}(\mathcal{T}, E))$ and in this case $X_{\bullet}=\left.X\right|_{A}: A \rightarrow A \cap E$, which is a Nijenhuis operator if $T^{X}$ vanishes.

\section{Acknowledgments}

Z. Chen would like to thank P. Xu and M. Stienon for the useful discussions and suggestions that helped him improving this work. Y.-H. Sheng gives his warmest thanks to L. Hoevenaars, M. Crainic, I. Moerdijk and C. Zhu for their help and useful comments during his stay in Utrecht University and Courant Research Center, Göttingen, where a part of work was done. Research partially supported by NSFC(10871007, 10911120391/A0109), Doctoral Fund. of MEC (20090001110006) and $\operatorname{CPSF}(20060400017)(20090451267)$; the third author financially supported by the governmental scholarship from China Scholarship Council. 


\section{References}

[1] A. Alekseev and Y. Kosmann-Schwarzbach, Manin Pairs and moment maps, J. Diff. Geom. 56 (2000), 133-165.

[2] Y. Bi and Y. Sheng, Higher analogues of Courant algebroids, arXiv:1003.1350, to appear in Science in China Series A: Mathematics.

[3] H. Bursztyn and M. Crainic, Dirac structures, moment maps and quasi-Poisson structures, Prog. Math. 232 (2007), 1-40.

[4] H. Bursztyn, G. Cavalcanti, M. Gualteri, Reduction of Courant algebroids and generalized complex structures, Adv. Math. 211 (2)(2007), 726-765.

[5] H. Bursztyn, D. Iglesias Ponte and P. Ševera, Courant morphism and moment maps, Math. Res. Lett. 16 (2009), no. 2, 215C232.

[6] J. Cervera, A.J. van der Schaft and A. Ba $\tilde{n}$ os, Interconnection of port-Hamiltonian systems and composition of Dirac structures, Automatica 43 (2007) 212-225.

[7] Z. Chen and Z.-J. Liu, Omni-Lie algebroids, J. Geom. Phys. 605 (2010), 799-808.

[8] Z. Chen, Z. Liu and Y. Sheng, E-Courant algebroids, Int. Math. Res. Not. Vol. 2010, No. 22, pp. 4334-4376.

[9] M. Crainic and R. L. Fernands, Secondary characteristic classes of Lie algebroids, Lect. Notes. Phys., 662(2005),157-176.

[10] T. Courant, Dirac manifolds, Trans. Amer. Math. Soc. 319(1990), 631-661.

[11] I. Ya. Dorfman, Dirac structures and integrability of nonlinear evolution equations, Wiley, Chichester, 1993.

[12] M. Gualtieri, Generalized complex geometry, PhD thesis, St John's College, University of Oxford, Nov. 2003.

[13] N. Hitchin, Generalized Calabi-Yau manifolds, Quart. J. Math. 54 (3) (2003), 281-308.

[14] C. Hofman and J.-S. Park, BV quantization of topological open membranes, Comm. Math. Phys. 249/2 (2004), 249-271.

[15] K. Kinyon and A. Weinstein, Leibniz algebras, Courant algebroids, and multiplications on reductive homogeneous spaces, Amer. J. math., 123(2001), 525-550.

[16] A. Kirillov, Local Lie algebras, Russian Math. Surveys, 31(1976), 55-76.

[17] Y. Kosmann-Schwarzbach and K. Mackenzie, Differential operators and actions of Lie algebroids, Contemp. Math., 315(2002), 213-233.

[18] C. Laurent, M. Stienon, and P. Xu, Non-abelian differential gerbes, Adv. Math. 220 (5) (2009), 1357-1427.

[19] A. Linchnerowicz, Les variétés de Jacobi et leurs algébres de Lie associées, J. Math. Pures et Appl., 57 (1978), 453-488.

[20] Z.-J. Liu, Some remarks on Dirac structures and Poisson reduction. Poisson Geometry Banach Center Publications, vol 51, 2000, 165-173.

[21] Z.-J. Liu, A. Weinstein and P. Xu, Dirac structures and Poisson homogeneous spaces, Comm. Math. Phys. 192 (1998), 121-144.

[22] Z.-J. Liu, A. Weinstein and P. Xu, Manin triples for Lie bialgebroids, J. Diff. Geom. 45(1997), 547-574.

[23] K. Mackenzie, General theories of Lie groupoids and Lie algebroids, Cambridge University Press, 2005.

[24] C. L. Rogers, 2-plectic geometry, Courant algebroids, and categorified prequantization, arXiv:1009.2975.

[25] D. Roytenberg, Courant algebroids, derived brackets and even symplectic supermanifolds, PhD thesis, UC Berkeley, 1999, arXiv:math.DG/9910078.

[26] D. Roytenberg, AKSZ-BV formalism and Courant algebroid-induced topological field theories, Lett. Math. Phys. (2007) 79: 143-159.

[27] P. Ševera and A. Weinstein, Poisson geometry with a 3-form background. Prog. Theor. 
Phys. Suppl. 144:145-154, 2001.

[28] D. J. Saunders, The Geometry of Jet Bundles, Cambridge University Press, Cambridge, 1989.

[29] Y. Sheng, On deformations of Lie algebroids, arXiv:1004.2959, to appear in Results in Math..

[30] Y. Sheng, Z. Liu and C. Zhu, Omni-Lie 2-algebras and their Dirac structures, J. Geom. Phys. 61 (2011), pp. 560-575.

[31] M. Stienon and P. Xu, Reduction of Generalized Complex Structures, J. Geom. Phys. 58(2008), 105-121.

[32] K. Uchino, Remarks on the definition of a Courant algebroid, Lett. Math. Phys. 60(2002): 171-175.

[33] K. Uchino, Courant brackets on noncommutative algebras and omni-Lie algebras, Tokyo J. of Math. 30 (1) (2007), 239-255.

[34] A. Wade, Conformal Dirac structures, Lett. Math. Phys. 53(2000), 331-348.

[35] A. Wade, Locally conformal Dirac structures and infinitesimal automorphisms, Comm. Math. Phys. 246 (2004), 295-310.

[36] A. Weinstein, Omni-Lie algebras, Microlocal analysis of the Schrodinger equation and related topics (Kyoto, 1999). No. 1176(2000), 95-102. 